\title{
PIONIC ANNIHILATION OF ANTIPROTONS STOPPED ON ${ }^{3} \mathrm{He}$
}

\author{
G. Bendiscioli, V. Filippini, A. Rotondi, P. Salvini and A. Venaglioni \\ Dipartimento di Fisica Nucleare e Teorica, University of Pavia and \\ INFN, Sezione di Pavia, Italy
}
S. Bossolasco, M.P. Bussa, L. Busso, L. Fava, L. Ferrero, A. Maggiora
D. Panzieri, G. Piragino, R. Piragino and F. Tosello
Istituto di Fisica Generale 'A. Avogadro', University of Turin and
INFN, Sezione di Torino, Italy

Yu. A. Batusov, I.V. Falomkin, G.B. Pontecorvo, A.M. Rozhdestvensky

M.G. Sapozhnikov and V.I. Tretyak

Joint Institute for Nuclear Research, Dubna, USSR

C. Guaraldo

Laboratori Nazionali di Frascati dell'INFN, Frascati, Italy

E. Lodi Rizzini

Dipartimento di Automazione Industriale, University of Brescia and

INFN, Sezione di Torino, Italy

A. Haatuft, A. Halsteinslid, K. Myklebost and J.M. Olsen

Physics Department, University of Bergen, Norway

F.O. Breivik, T. Jacobsen and S.O. Sørensen,

Physics Department, University of Oslo, Norway

F. Balestra

Dipartimento di Scienze Fisiche, University of Cagliari and

INFN, Sezione di Cagliari, Italy

A. Zenoni*)

CERN/EP, Geneva, Switzerland

\begin{abstract}
The pionic annihilation of antiprotons stopped on ${ }^{3} \mathrm{He}$ nuclei in a self-shunted streamer chamber exposed to the antiproton beam of LEAR is studied. The data concern charged particle multiplicity distributions, branching ratios for different final states, the probability of final state interaction, $\pi^{-}, \pi^{+}, p$ and $d$ momentum spectra, like and unlike pion angular correlations, $\pi$ and other charged particles angular correlations. The comparison of the ${ }^{3} \mathrm{He}$ data with those obtained on ${ }^{1} \mathrm{H},{ }^{2} \mathrm{H}$ and ${ }^{4} \mathrm{He}$ does not reveal relevant effects due to the increase of the nucleon number, the small differences can be seen as due to a weak final state interaction.
\end{abstract}

(Submitted to Nuclear Physics A)

*) On leave of absence from INFN, Sezione di Pavia, Italy. 


\section{INTRODUCTION}

The simplest picture of the antiproton-nucleus annihilation process at low energy considers a three step process $[1,2,3]: 1)$ the antiproton annihilates on a single nucleon producing mesons as in the free space case; 2 ) the created mesons interact with the nucleons in the residual nucleus producing a cascade of binary collisions and decays according to their free space cross sections (except for Pauli blocking effects); in this stage a fraction of pions is absorbed and some fast nucleons are ejected as a result of two-body interactions; 3 ) an excited nucleus survives after this fast ejecton process, which losses its energy via different mechanisms, depending on the value of the excitation energy.

However, the existence of some nucleons in the vicinity of the annihilation point in the antiproton-nucleus interaction opens the possibility of unusual annihilation processes involving more than one nucleon, which do not reduce to the single-nucleon annihilation followed by meson rescattering[4,5,6,7,8,9,10]. The annihilation on few nucleons can be interpreted in terms of quark degrees of freedom. For instance, if two nucleons are close enough, an antiquark in the antiproton could annihilate with a quark in one nucleon bag, while the other antiquarks annihilate with the quarks of a second nucleon bag. The antiquarks in this process can be considered as being partially delocalized, since they reach two near bags $[9,11]$.

The study of the annihilation of antiprotons on the lightest nuclei such as ${ }^{2} \mathrm{H},{ }^{3} \mathrm{He}$ or ${ }^{4} \mathrm{He}$ is interesting for more reasons. First of all, it is possible to obtain information on the antiproton-neutron interaction which is hardly obtainable from the antineutron-proton annihilation due to the bad quality of the present antineutron beams. Secondly, it is interesting to study the effects of the final state interactions (FSI) of the annihilation mesons. These effects can be identified more clearly in the case of the annihilation on few nucleon systems. Thirdly, it is of great importance to look for unusual annihilation processes.

The main goal of studying antiproton-neutron annihilation is to obtain information on the isospin structure of the antinucleon-nucleon interaction. The knowledge of the isospin dependence is important to understand what is the antiproton annihilation at low energies. It might be mainly a phase-space governed process without any strong spin or isospin dependence [12] or some nontrivial "selection rules" might exist as a reflection of the underlying quark dynamics [13]. At low momenta (say below $600 \mathrm{MeV} / \mathrm{c}$ ) antinucleon-nucleon and antinucleon-nucleus data indicate that the ratio $\mathrm{R}=\sigma_{\mathrm{pn}}^{\mathrm{a}} / \sigma_{\mathrm{pp}} \mathrm{p}_{\mathrm{p}}$ is a bit lower than one; that is, the annihilation cross section in flight in the $\mathrm{T}=0$ isospin state is a bit higher than in the $\mathrm{T}=1$ state, in agreement with many optical models (for reviews see $[14,15])$. A similar result was found also at rest on liquid deuterium $(R \approx 0.8)[16,17]$ where the annihilation occurs mainly from S-levels. On the contrary, a quite different result was found at rest on gaseous ${ }^{3} \mathrm{He}$ and ${ }^{4} \mathrm{He}$ targets $(R=0.5)[15,18]$, where the annihilation occurs mainly from $P$ - and D- levels [19], revealing a strong dependence of $R$ on the angular momentum. This is not accounted for in the framework of some optical potentials [20,21]. In these models the isospin independent annihilation potential was used, where the only isospin dependence comes from the G-parity conjugated meson exchange parts of the NN-potential. Therefore the difference between the experimental value of $R$ and the theoretical one may indicate the need of introducing obligatory the isospin dependence into the annihilation potentials. So, it is interesting to investigate in detail what happens in the case of antiproton annihilation on gaseous ${ }^{3} \mathrm{He}$ compared with the liquid deuterium case.

The effects of FSI in the antiproton-nucleus annihilation may be responsible of the unusual high yield of $\Lambda^{\circ}$ hyperons which was observed recently in the annihilation on Ta at $4 \mathrm{GeV} / \mathrm{c}[22]$ as well as in that on $\mathrm{Ne}{ }^{[23]}$ and ${ }^{4} \mathrm{He}{ }^{[24]}$ at $600 \mathrm{MeV} / \mathrm{c}$. In its turn, this high yield may explain the large probability of heavy hypernuclei formation which was observed in the antiproton-nucleus annihilation [25]. The exact identification of the meson FSI effects may help in understanding whether these phenomena are due to ordinary rescattering of kaons or whether other mechanisms such as the $B=1$ fireball formation [26] or the supercooled quagma evaporation ${ }^{[4]}$ are also relevant.

As an example of the different hypotheses of unusual antiproton-nucleus annihilation, we recall that of Cugnon et al.[5,9]. They speculated about the possibility that a fireball formed by antinucleon-nucleon annihilation catches a neighbouring nucleon (or more nucleons) creating an object with baryon number $\mathrm{B}=1$ (or $\mathrm{B}>1$ ). This fireball may evaporate according the same thermodynamical laws which were found to be useful in the description 
of the ordinary antinucleon-nucleon annihilation. However, the specific baryon content of these fireballs changes the characteristics of the emitted particles in comparison with the $B=0$ fireball evaporation. The most distinctive feature is the increasing of strange particle yield. But multiplicities and momentum spectra (particularly in the high momentum region) are also varied. It was predicted that the $\mathrm{B}=1$ fireball may be formed in $10 \%$ cases of antiproton annihilation on ${ }^{3} \mathrm{He}$ [9].

This paper contributes to these fields with an experimental study of the annihilation into pions at rest on ${ }^{3} \mathrm{He}$. In the antiproton-nucleus interaction at rest, the annihilation is the last step of a chain of processes, which begins with the capture of a very slow antiproton on an atomic orbit and proceeds with the de-excitation of the atom down to an atomic level where the energy and the width are affected by the nuclear field. The annihilation occurs on the far periphery of the nucleus, so it should have features very similar to those on a free nucleon; moreover the final state interaction is smaller than that for annihilation in flight [27].

No prediction on the annihilation at rest on ${ }^{3} \mathrm{He}$ exists, the few available studies being limited to the momentum region above $300 \mathrm{MeV} / \mathrm{c}[9,28]$.

In order to put in evidence some features dependent on the different numbers of nucleons, we will discuss our data by comparing them with similar data on ${ }^{1} \mathrm{H},{ }^{2} \mathrm{H}$ and ${ }^{4} \mathrm{He}$. This comparison is the final goal of our paper.

The data were obtained with a self-shunted streamer chamber in a magnetic field exposed to the antiproton beam of the LEAR facility at CERN. They concern charged particle multiplicity distributions, branching ratios of different reactions, the probability of the FSI effects, $\pi^{-}, \pi^{+}, \mathrm{p}$ and $\mathrm{d}$ momentum spectra, like and unlike pion angular correlations, $\pi$ and other charged particle angular correlations.

The present study adds to a previous work on the annihilation cross section on ${ }^{3} \mathrm{He}$ at $200 \mathrm{MeV} / \mathrm{c}^{[29]}$ and completes previous works at rest devoted to find out the ratio between the probability of annihilation on a single neutron and that on a single proton $[15,18,30]$. Some data on ${ }^{4} \mathrm{He}$ (the angular correlations and the $\pi \mathrm{N}$ charge exchange) are given here for the first time.

\section{EXPERIMENTAL CONDITIONS}

The experimental apparatus has been described previously in detail [31], here we only recall its chief features. The self-shunted streamer chamber, filled with ${ }^{3} \mathrm{He}$ at $1 \mathrm{~atm}$, served at the same time as a target and detector. The volume of the chamber was $70 \times 90 \times 18 \mathrm{~cm}^{3}$ and the magnetic field was $0.4 \mathrm{~T}$. The thickness of the target was $15 \mathrm{mg} / \mathrm{cm}^{2}$. The trigger for the high voltage pulse generator of the chamber was provided by a hodoscope of thin scintillation counters placed along the beam, in front of the chamber. The antiproton momentum of the LEAR beam was $105 \mathrm{MeV} / \mathrm{c}$. Taking into account the energy losses through the beryllium end wall of the vacuum line of LEAR, the scintillation counters of the trigger and through the entrance window of the chamber, the antiproton momentum at the beginning of the sensitive volume was spread around $61 \mathrm{MeV} / \mathrm{c}(2 \mathrm{MeV})$. The antiproton stop points took place about the center of the chamber. The great majority of the annihilations occured at rest, the probability of those in flight being less than $10^{-3}$ [32]. The events were photographed by two cameras, with one event per photo. For achieving well-localized bright tracks in the chamber about $0.3 \%$ of iso- $\mathrm{C}_{4} \mathrm{H}_{10}$ was included in the ${ }^{3} \mathrm{He}$ gas filling of the chamber.

Since the total charge of the final state in antiproton $-{ }^{3} \mathrm{He}$ annihilation equals +1 , the prong multiplicity distribution contains only odd-prong events as it is shown in Tab. 3.1. Nevertheless, we observed also some even-prong events, which are due to annihilations on the $\mathrm{C}_{4} \mathrm{H}_{10}$ admixture. Annihilation on $\mathrm{H}$ only produces events with an even number of prongs and annihilation on $\mathrm{C}$ produces both even prong and odd prong events. The latter may be confused with events on ${ }^{3} \mathrm{He}$ if the number of the positive prongs exceeds that of the negative prongs by one. We estimated a possible contribution of spurious events with an odd number of charged prongs to be less than about $1.1 \%$ of the detected events [29].

It must be stressed that a streamer chamber operating at low pressure represents a very good instrument for studying charged-particle multiplicities. In fact, for instance, the tracks of a $250 \mathrm{keV} \alpha$-particle or of a $160 \mathrm{keV}$ proton are $1 \mathrm{~cm}$ long in the chamber and are quite visible. 


\section{ANTIPROTON-3He ANNIHILATION.}

Antiproton passing through the gas target are slowed down by inelastic collisions until they stop and are captured in outer Bohr orbits of ${ }^{3} \mathrm{He}$ atoms in states with high angular momentum. An antiprotonic atom losses angular momentum and energy by Auger and radiative transitions until the antiproton falls into an orbit where the strength of the nuclear field is sufficient for annihilation to occur. In the helium gas at one atmosphere and room temperature antiprotons annihilate mostly from P-states (49\%) and D-states (43\%) [19].

We notice that we have not separated pions from kaons, so our distributions include both types of particles. However, kaons are present only in a few percent of the cases.

If in the annihilation process only the antiproton-nucleon annihilation is effective and the final state interaction (FSI) is not, the antiproton-proton annihilation produces an even number of charged pions and a ${ }^{2} \mathrm{H}$ nucleus (or an unbounded proton-neutron pair) and the antiproton-neutron annihilation produces an odd number of charged pions and two protons. Hence, in all cases we have an odd number of prongs. The annihilation on p produces, on the average, $1.5 \pi^{-}, 1.5 \pi^{+}$and $2 \pi^{\circ}$; the annihilation on $n$ produces, on the average, $1 \pi^{+}$, $2 \pi^{-}$and $2 \pi^{\circ}[11]$.

FSI may break the ${ }^{2} \mathrm{H}$ nucleus or, through the $\pi$-nucleon absorption and charge exchange reactions $\left(\pi^{-} p<->\pi^{0} n, \pi^{0} p<->\pi^{+} n\right)$ or the $\pi d$-absorption may change the primary number of charged pions and charged heavy prongs. An important consequence of the charge exchange is that the antiproton-proton annihilation events may assume the features of events due to antiproton-neutron annihilations and vice versa, as it affects the relative number of heavy and light particles emitted. This fact is illustrated by the examples in Tab. 3.1. We see that, in general, there is no distinction between events due to annihilation on proton and on neutron on the basis of the number of heavy and light particles. In spite of this, it is possible to evaluate the probabilities of annihilation on single proton and on single neutron, owing to a compensation between the numbers of exchanged events, as it is shown in Refs. 15 and 18. follows:

On the basis of the different residual nucleons, all the reactions can be collected as

$$
\begin{aligned}
\overline{\mathrm{p}}^{3} \mathrm{He} \rightarrow & 2 \mathrm{n}+(\mathrm{k}+1) \pi^{+}+\mathrm{k} \pi^{-}+\mathrm{h} \pi^{\mathrm{o}} \\
& \mathrm{p}+\mathrm{n}+\mathrm{k} \pi^{+}+\mathrm{k} \pi^{-}+\mathrm{h} \pi^{0} \\
& \mathrm{~d}+\mathrm{k} \pi^{+}+\mathrm{k} \pi^{-}+\mathrm{h} \pi^{0} \\
& 2 \mathrm{p}+\mathrm{k} \pi^{+}+(\mathrm{k}+1) \pi^{-}+\mathrm{h} \pi^{0}
\end{aligned}
$$

The reactions (1) and (3) imply antiproton-proton annihilation only. The reactions (2) and (4) may arise from antiproton-proton or antiproton-neutron annihilations (see Tab. 3.1).

The reactions can be ordered usefully also according to the charged particle multiplicity $(M)$, the $\pi^{-}$multiplicity $\left(n_{\pi^{-}}\right)$and the number of heavy prongs $\left(n_{h}\right)$. Note that $M=2 n_{\pi^{-}}-1$, $M=1,3,5, \ldots$ and $n_{\pi^{-}}=0,1,2, \ldots$. Reaction (1) produces no heavy prong; reactions $(2-3)$ produce one heavy prong and reaction (4) produces two heavy prongs.

\section{PRONG MULTIPLICITIES AND BRANCHING RATIOS}

In this section we estimate the branching ratios for the reactions (1-4) and the multiplicity distributions of the various final particles. To this aim, the main problem is the identification of these particles.

\subsection{Particle identification}

We have observed a maximum number of prongs per event equal to 9 , corresponding to a maximum number of negative particles equal to 4 .

The negative particles are distinguished from the positive ones by the curvature sign. They are mostly pions and secondary kaons (present in about $4.6 \%$ of the events on ${ }^{1} \mathrm{H}^{[33]}$ ). The positive particles may be pions, kaons (few percent), protons and deuterons. They can be identified by considering features of the single tracks (minimum reduced $\chi^{2}$ value given by the geometrical reconstruction program for different mass hypotheses; relation between 
stopping power and blackness or streamer density of the tracks) and features of the whole event (charge and baryonic number conservation). The identification criteria are described widely in Ref. 34 for the annihilation on ${ }^{4} \mathrm{He}$. The present case is simpler due to the smaller number of possible final particles.

Concerning the use of the ionization (blackness of the tracks), we recall that pions cannot be distinguished from kaons above $350 \mathrm{MeV} / \mathrm{c}$ and from protons above $550 \mathrm{MeV} / \mathrm{c}$ (see Fig. 4.1); protons cannot be distinguished from deuterons below $200 \mathrm{MeV} / \mathrm{c}$, as both produce tracks with the same high ionization effects. So the ability of our apparatus in identifying different particles depends on their momentum. This inefficiency is overcome partially by considering the baryonic number conservation. Indeed (see reactions (1-4)), at most two protons may be present among the final products and the presence of a deuteron excludes that of protons and viceversa.

We have identified the particles as indicated in Tab. 4.1. In some cases the identification is univocal, in other cases the identification is only partial; for example, we can exclude that a prong is due to a deuteron, but we cannot decide between meson and proton, and so on. In some cases no identification is possible. Beside by the ionization effects, the identification is impeded also by stereoscopic effects and by geometrical limitations depending on the directions of the tracks with respect to the magnetic field direction (see also later on).

\subsection{Event identification}

On the basis of the mass identification, we can recognize the events according to the reactions (1-4): we will denote these events by the number $n_{h}$ of heavy particles. Due to the impossibility of identifying the mass of each prong, there is a number of events identified only partially and a number of events not identified at all. Summarizing, we have grouped the events according to the number of heavy prong as follows (see Tabs. 4.2 and 4.3): $n_{h}=0$, $n_{h}=1, n_{h} \leq 1 ; n_{h} \geq 1 ; n_{h}=2 ; n_{h}=0,1,2 . n_{h} \leq 1$ indicates events with a number of heavy prongs uncertain between 0 and 1 , but not higher than $1 . n_{h} \geq 1$ indicates events with a number of heavy prongs equal to 1 or 2 , but not $0 . n_{h}=0,1,2$ indicates events where it is not possible decide whether heavy prongs are present or absent. The above sets of events are mutually exclusive.

Tab. 4.2 shows that the identification procedure is more efficient for events with low multiplicity as the identified events (i.e., the sum of those with $n_{h}=0, n_{h}=1, n_{h} \leq 1, n_{h}=2$ ) with multiplicity 3 are more than those with multiplicity 5 , differently from the case of the complete set of events (see Tab. 4.4 , column b).

Among the effects hindering the mass identification, the stereoscopic and geometrical ones are statistical, as the tracks are directed at random in space. Assuming that these effects are dominant, we can evaluate the relative probability of the reactions (1-4), although not all the events are identified. With reference to Tabs. 4.2 and 4.3, we proceed as follows.

Let us consider events with a given multiplicity and neglect for the momentum the events with $n_{h}=0,1,2$. We indicate with $n(0), n(1), n(2)$ the number of identified events with $n_{h}=0, n_{h}=1$ and $n_{h}=2$ respectively.

The events with zero heavy prongs are in part identified $(n(0))$ and in part included into those with $n_{h} \leq 1(n(\leq 1))$. We assume that the fraction of the latter events is given by

$$
\frac{n(0)}{n(0)+n(1)}
$$

Hence the total number of events without heavy prongs is

$$
n^{\prime}(0)=n(0)+n(\leq 1) \frac{n(0)}{n(0)+n(1)}
$$

The events with one heavy prongs are in part identified (n(1)), in part included into those with $n_{h} \leq 1$ and in part included into those with $n_{h} \geq 1$. For the second ones we assume that their fraction is 


$$
\frac{n(1)}{n(0)+n(1)}
$$

so that the number of events with $n_{h}=1$ becomes at a first step

$$
n^{\prime \prime}(1)=n(1)+n(\leq 1) \frac{n(1)}{n(0)+n(1)}
$$

For the third ones we assume that their fraction is

$$
\frac{\mathrm{n}^{\prime \prime}(1)}{\mathrm{n}^{\prime \prime}(1)+\mathrm{n}(2)}
$$

Hence, at a second step, the total number of events with $n_{h}=1$ is

$$
n^{\prime}(1)=n(1)+n^{\prime \prime}(1)+n(\geq 1) \frac{n^{\prime \prime}(1)}{n^{\prime \prime}(1)+n(2)}
$$

The events with $n_{h}=2$ are in part identified and in part included into those with $n_{h}>1$. Their total number is

$$
n^{\prime}(2)=n(2)+n(\geq 1) \frac{n(2)}{n^{\prime \prime}(1)+n(2)}
$$

Finally, we assume that the number of the unidentified events $\left(n_{h}=0,1,2\right)$ is divided proportionally to $n^{\prime}(0), n^{\prime}(1)$ and $n^{\prime}(2)$. So the final numbers of events with a given multiplicity are

$$
\begin{aligned}
& n_{\text {tot }}(0)=n^{\prime}(0)+n(0,1,2) \frac{n^{\prime}(0)}{n^{\prime}(0)+n^{\prime}(1)+n^{\prime}(2)} \\
& n_{\text {tot }}(1)=n^{\prime}(1)+n(0,1,2) \frac{n^{\prime}(1)}{n^{\prime}(0)+n^{\prime}(1)+n^{\prime}(2)} \\
& n_{\text {tot }}(2)=n^{\prime}(2)+n(0,1,2) \frac{n^{\prime}(2)}{n^{\prime}(0)+n^{\prime}(1)+n^{\prime}(2)}
\end{aligned}
$$

Considering the numbers in Tab. 4.2, we obtain the percentages of events with $n_{h}=0, n_{h}=1$ and $n_{h}=2$ for each multiplicity value, which are given in Tab. 4.4. Summing up for each value of $n_{h}$, we obtain the probabilities of reaction (1) $\left(n_{h}=0\right)$, reactions (2) plus (3) and reaction $(4)\left(n_{h}=2\right)$, respectively. The probabilities for the reactions (2) and (3), separately, are obtained considering that, among the events with $n_{h}=1$, those with one proton and those with a deuteron are in the ratio 135/210 (see Tab. 4.3). All the probabilities are given in Tab. 4.4.

Part (c) of this table gives the $\pi^{-}$multiplicity distributions for the different reactions, which are obtained from part (a) normalizing to 100 the percentages for each value of $n_{h}$. Tab. 4.4 gives also the mean values of $\pi^{-}$, all prongs and heavy particles for the different reactions.

Tab. 4.4 allows us to carry out also the $\pi^{+}$multiplicity distribution. Indeed, for each value of $M$, the number of $\pi^{+}$is strictly related to those of $\pi^{-}$and of heavy prongs, as is shown in tab. 4.5. So, the percentage of events with zero $\pi^{+}$is given by the sum of the percentages of events with $\left(n_{h}=1, n_{\pi^{-}}=0\right)$ and with $\left(n_{h}=2, n_{\pi^{-}}=1\right)$ and so on. Considering Tab. 4.4, part (a), one obtains the $\pi^{+}$multiplicity distribution given in Tab. 4.6 and the correspondent mean number of $\pi^{+}$per event.

According to Ref. 15, the sum of the multiplicity distributions of the events with $n_{h}=0$ 
and $n_{h}=1$ (part (a) of Tab. 4.4) gives the multiplicity distribution for annihilations on proton and the multiplicity distribution of the events with $n_{h}=2$ represents the multiplicity distribution for annihilations on neutron. These distributions normalized to 100 are given in Tab. 4.7. According to Ref. 15 , the ratio $R=2(P(2) /(P(0)+P(1))=0.465 \pm 0.041$ is the ratio between the probabilities of annihilation on a single neutron and on a single proton in $\mathrm{P}$ - and $D$ - waves. The multiplicity distributions for annihilation on single nucleon and the value of $R$ found here coincide with those obtained previously in Ref. 15 with a somewhat different procedure.

\subsection{Comments}

In Tab. 4.7 the $\pi^{ \pm}$multiplicity distribution for antiproton-proton and antiproton-neutron annihilations on ${ }^{3} \mathrm{He}$ are compared with those obtained from ${ }^{1} \mathrm{H},{ }^{2} \mathrm{H}$ and ${ }^{4} \mathrm{He}$. We see that the mean numbers of $\pi^{+}$and of $\pi^{-}$obtained from the different nuclei as well as, at a first glance, the multiplicity distributions are equal within the errors.

However the distributions display some differences, although small. Let us consider the annihilation on proton. We see that the $\pi^{-}$distributions varies regularly from ${ }^{1} \mathrm{H}$ to ${ }^{4} \mathrm{He}$. Specifically, the percentage for $n_{\pi^{-}}=1$ increases and that for $n_{\pi^{-}}=2$ decreases as the mass number increases. Anyway, we recall that the multiplicity distributions for ${ }^{3} \mathrm{He}$ and ${ }^{4} \mathrm{He}$ include the charged kaons.

We note that the features of the multiplicity distributions are interesting from more points of view. They are related, for instance, to the isospin dependence of the antinucleon-nucleon annihilation process, to the final state interactions and to the multinucleon annihilation $[2,3,36]$. Here, we consider the first two points. Bearing in mind (see Sect. 1) that the antiproton annihilation in ${ }^{3} \mathrm{He}$ gas proceeds mainly from $\mathrm{P}$ - and $\mathrm{D}$-states whereas the annihilation in liquid hydrogen takes place from S-levels, one may regard the results of Tab. 4.7 as an indication that the average annihilation meson multiplicities are not very dependent on the angular momentum. Really, this statement may be corrected only for multiplicities averaged over reaction channels with different numbers of pions, because for the separate channels the angular momentum dependence must exist. For example, the final state $\pi^{+} \pi^{-} 2 \pi^{\circ}$ is forbidden in the antiproton-proton annihilation from ${ }^{1} S_{o} ; 2 \pi^{-} \pi^{+} \pi^{\circ}$ is forbidden in the antiproton-neutron P-states, etc. [37].

The similarity of the meson multiplicity distributions for the lightest nuclei seems to indicate a negligible absorption and charge exchange (CEX) of the annihilation mesons. Really, this is true as it concerns the absorption, as the mean numbers of $\pi^{-}$and $\pi^{+}$are independent of the mass number, but it is not quite so for the charge exchange. First of all, there are some systematical trends in the antiproton-proton multiplicities, as stressed previously. But a more clear and convincing demonstration of the FSI effects is given by the results of Tab. 4.4 concerning the multiplicities of events without heavy prongs, i.e. without protons or deuterons. These events are produced on ${ }^{3} \mathrm{He}$ when the annihilation takes place on a proton and, after that, the annihilation mesons convert the other proton into a neutron. In principle, reactions of the following type are possible:

$$
\begin{aligned}
& \pi^{-}+p->\pi^{0}+n \\
& \pi^{0}+p->\pi^{+}+n \\
& \pi^{-}+d->n+n \\
& K^{-}+p \rightarrow \bar{K}^{o}+n
\end{aligned}
$$

However the probability of the reactions (c) and (d) is much smaller than that of (a) and (b) (charge exchange reactions), so we assume that only the latter produce 0 prong events.

The first column of Tab. 4.4, part (c) shows that the $\pi^{-}$multiplicity distribution of the 0 -prong events does not follow the multiplicity distribution of the annihilation on free proton. The features of this distribution can be explained as follows.

With reference to Tab. 4.5 , if $n_{h}$ goes from 1 to zero due to $\pi^{-}$CEX, then M decreases by $2, n_{\pi^{-}}$decreases by 1 and $n_{\pi}+$ is unchanged; if $n_{h}$ goes to zero due to $\pi^{\circ}$ CEX, then $M$ and $n_{\pi^{-}}$do not change and $n_{\pi}+$ increases by 1 . That is, $\pi^{\circ}$ CEX contributes to 0 -prong events with the same $M$ and $n_{\pi^{-}}$as the original 1-prong events; $\pi^{-}$CEX contributes to 0 -prong events with lower multiplicities. On the other side, Tab. 4.4 shows that the dominant $n_{h}=1$ channels are those with $\left(n_{\pi^{-}}=1, n_{\pi^{+}}=1\right)$ and $\left(n_{\pi^{-}}=2, n_{\pi^{+}}=3\right)$. So 0 -prong events with 
$\mathrm{n}_{\pi^{-}}=0$ arise only from 1-prong events with $\left(\mathrm{n}_{\pi^{-}}=1, \mathrm{n}_{\pi^{+}}=1\right)$, those with $\mathrm{n}_{\pi^{-}}=2$ arise mainly from events with $\left(n_{\pi^{-}}=2, n_{\pi^{+}}=3\right)$ and those with $n_{\pi^{-}}=1$ from both the dominant $\mathrm{n}_{\mathrm{h}}=1$ channels. The total probability of 0 -prong event is $3.75 \%$ (see Tab. 4.4).

In conclusion, from these considerations one may estimate that the probability for annihilation pions being involved in CEX reaction of (a) or (b) type is around 2-3\%. Taking into account three possible pion charged states and also the annihilation on the neutron one may estimate that the charge-exchange reactions of all mesons on both nucleons may reach $10-15 \%$ of all the annihilation probability on ${ }^{3} \mathrm{He}$.

Comments similar to those made on the $\pi^{-}$multiplicity distributions for annihilation on proton could be made for the annihilation on neutron, but in this case the errors are quite large.

As a final remark, we note that $\left\langle\pi^{-}\right\rangle$and $\left\langle\pi^{+}\right\rangle$from ${ }^{1} \mathrm{H}$ and ${ }^{2} \mathrm{H}$ in Tab. 4.7 were obtained with liquid targets and are related to a mean value of charged pions $<n_{\pi} \pm>$ equal to $3.05 \pm 0.02^{[17]}$. This value is in agreement with Ref. 16 too. Recently, with a gaseous ${ }^{2} \mathrm{H}$ target has been obtained a higher value, namely $\left\langle n_{\pi} \pm\right\rangle=3.26 \pm 0.05[38]$. The value obtained from deuterium is the mean over the mean values of $\pi$ and of $\pi^{+}$produced on neutron and on proton. If $R$ is the ratio between the probabilities of annihilation on neutron and on proton, we have

$$
\left\langle\mathrm{n}_{\pi^{ \pm}}\right\rangle=\frac{1}{1+\mathrm{R}}\left[\left(\left\langle\pi^{+}\right\rangle+\left\langle\pi^{-}\right\rangle\right)_{\text {proton }}+\mathrm{R}\left(\left\langle\pi^{+}\right\rangle+\left\langle\pi^{-}\right\rangle\right)_{\text {neutron }}\right]
$$

For liquid deuterium $R=0.749 \pm 0.018[17]$ and the values of $\left\langle n_{\pi^{-}}\right\rangle$and $\left\langle n_{\pi^{+}}+\right\rangle$are given in Tab. 4.7. One obtains just $\left\langle n_{\pi} \pm>=3.05\right.$. To obtain $\left\langle n_{\pi} \pm>=3.26\right.$, $R$ and/or $\left\langle\pi^{+}>\right.$should have values quite different from the previous ones. Note that $\left\langle\mathrm{n}_{\pi} \pm\right\rangle$ approaches $\left(<\pi^{+}>+<\pi^{-}>\right)_{\text {neutron }} \approx 3.152<3.26$ when $\mathrm{R}>>1$. Moreover, a higher value of $<\pi^{+}>\left(<\pi^{-}>\right)$ should imply multiplicity distributions where the higher multiplicity channels have higher weigths compared to those in Tab. 4.7.

\section{MOMENTUM DISTRIBUTIONS}

\subsection{Efficiency in momentum measurement}

The streamer chamber allows the tracks to be detected isotropically, but it does not allow the momenta to be measured isotropically (see Fig. 5.1). Really, the magnetic field is more effective in determining the curvature of the tracks in a plane perpendicular to its own direction and is totally inefficient along it; moreover, the sensitive volume has its largest dimensions in this plane and the smallest one in the direction perpendicular to it. So, considering tracks with the same momentum, their sagittas, which determine the measurement of the momentum, are more remarkable for tracks close to the above plane than for those close to the magnetic field direction.

Moreover, among tracks with the same dip angle, the measurement of low momenta is more favourite than that of high momenta, due to the longer sagittas. This is put in evidence by Fig. 5.2 where the momentum distribution of all $\pi^{-}$is compared with the momentum distribution of the $\pi^{-}$with dip angle between $\pm 30^{\circ}$. One sees that the distribution of all $\pi^{-}$is richer of $\pi^{-}$with lower momenta. The distortion decreases as the dip angle decreases. We have verified that for momenta below $1 \mathrm{GeV} / \mathrm{c}$ it disappears for dip angles smaller than $30^{\circ}$.

\section{$5.2 \pi^{-}$momentum spectra}

The overall $\pi^{-}$momentum spectrum is shown in Fig. 5.2. Its behaviour is determined mainly by the spectrum of the $\pi^{-}$emitted in annihilations with $M=5\left(n_{\pi^{-}}=2\right)$, as it is shown in Fig. 5.3.

In Fig. 5.4 the $\pi^{-}$spectra for events with a deuteron (annihilations on p) and with two $\mathrm{p}$ (annihilations on $\mathrm{n}$ or $\mathrm{p}$ with $\pi \mathrm{N}$ charge exchange effects) are shown. One sees that, within the limits of the present statistics, all the spectra are similar.

In Fig. 5.5 the spectrum of the pions emitted in events without heavy prongs is shown. It is strongly peaked at low momenta.

\subsection{Momentum spectra of positive particles}


The momentum spectrum of all the positive particles is shown in Fig. 5.6, while the different spectra of the identified pions, protons and deuterons are shown in Fig. 5.7. This figure shows also the spectrum of unidentified heavy particles and that of positive particles completely unidentified.

In spite of the impossibility of separating completely $\pi^{+}$from the other particles, particularly in the high momentum region, the $\pi^{+}$spectrum is very close to that of the $\pi^{-}, a$ part a lack of particles in the high momentum tail (see Fig. 5.8). Correspondingly, a tail rich of particles appears in the spectrum of the unidentified particles. We may conclude that also the spectra of the protons and of the deuterons are not strongly affected by the limitations in their identification. These spectra develop mainly below $400 \mathrm{MeV} / \mathrm{c}$ with a peak between 40-80 MeV/c. Nothing can be said about the high momentum tails.

\subsection{Comments}

In Figs. 5.9 and 5.10 the $\pi^{-}$momentum distribution from ${ }^{3} \mathrm{He}$ is compared with those from ${ }^{1} \mathrm{H}, \mathrm{n}\left({ }^{2} \mathrm{H}\right),{ }^{4} \mathrm{He}$ and ${ }^{20} \mathrm{Ne}$. The ${ }^{3} \mathrm{He}$ spectrum is egual to the ${ }^{4} \mathrm{He}$ one and very close to the ${ }^{1} \mathrm{H}$ one, but shifted a bit towards the small momentum region. This seems to be according with the fact that the annihilation process at rest in $\mathrm{He}$ is dominated by the annihilation on proton ( $R=0.46$, see sect. 4.2$)$ and the $\pi N$ absorption and rescattering are weak. The different bond and number of nucleons in the two nuclei seems to affect negligibly the annihilation process, at least as it concerns the pion emission. We recall, also, that in the annihilation in flight $(200 \mathrm{MeV} / \mathrm{c})$, the cross sections for annihilation on ${ }^{3} \mathrm{He}$ and ${ }^{4} \mathrm{He}$ are equal due a compensation between the larger size of ${ }^{3} \mathrm{He}$ and the larger number of nucleons in ${ }^{4} \mathrm{He}$ [29]. To stress the increasing role of a multinucleon system in the annihilation on nuclei at rest, in fig. 5.10 the $\pi^{-}$spectrum from ${ }^{20} \mathrm{Ne}$ is shown. It is shifted again more towards the small momentum region.

The ${ }^{3} \mathrm{He} \pi^{-}$spectrum can be fitted by a thermodynamics distribution of maxwellian type $d N / d p \approx\left(p^{2} / E\right) \exp (-E / T)[11,39]$ with $\mathrm{T}=135.4 \pm 2.7 \mathrm{MeV}$ (see Fig. 5.11), which is very close to the values found on other light nuclei (see Tab. 5.1). On the first glance, we have not seen any depletion in the low-energy part of the pion momentum spectra as was predicted in Ref. [42]. It must be noted that the streamer chamber provide a unique possibility to investigate just the low energy part of the spectrum.

\section{ANGULAR CORRELATIONS}

\section{1. $\pi \pi$ correlations}

The interest of the angular correlations deals with the so called GGPL effect. As pointed out firstly by Goldhaber et al. [43] studying the antiproton-proton annihilation, there exists a charge correlation effect in the distribution of the opening angles of the pion pairs. The distributions of the angle included by pairs of pions with the same and opposite charge are quite different. According to the phase space calculation, the opening angle distributions are peaked at $180^{\circ}$, but the observed like pion distribution is less peaked than the phase space one and the unlike pion distribution is more peaked[44]. It has been pointed out by a number of authors (Refs. 44 and 45 and the references quoted therein) that the correlation between incoherently emitted like particles is related to the spatial and time structure of the emitting source. So one may hope to put in evidence some differences between the annihilation mechanisms on free nucleons and on nuclei by comparing angular correlations obtained from the two systems.

Fig. 6.1 display the $\cos \theta$ distributions for charged pion pairs with like $\left(\pi^{ \pm \pm}\right)$and unlike $\left(\pi^{+} \pi^{-}\right)$charge, $\theta$ being the angle between two pions of the same event in the c.m. system (which in our case coincides with the laboratory system). The main features of these distributions can be synthetized by the quantities $\gamma^{\#}, \gamma^{+}$defined by

$$
\gamma=\frac{\text { Number of pairs with } \theta>90^{\circ}}{\text { Number of pairs with } \theta<90^{\circ}}
$$

The values of $\gamma$ are shown in tab. 6.1. The phase space calculation predicts $\gamma>1$ [44].

We have considered separately the sets of the events with production of zero, one and two heavy prongs, where all $\pi^{+}$and $\pi^{-}$are identified and those where the particles are more 
or less unidentified. $\pi^{-}$, which are the best identified particles, produce $\gamma^{-}$values very close each others independently of the event set. $\gamma^{++}$values are more spread and, on the average, lower, but the $\pi^{+}$identification depends, in some cases, on the track direction and momentum. $\gamma^{+-}$values for the identified events are very close each others. In Tab. 6.1 we give the values of $\gamma^{ \pm+}$and $\gamma^{+-}$for all the events and for the identified ones (that is, those with zero, one and two heavy prongs). Also the values of $\gamma^{-}$are given separately.

The main characateristics of the distributions are the following: 1) the unlike pion pair distributions are peaked remarkably at $\theta=180^{\circ}$, which reflects in a high value of $\gamma(\sim 2)$. Within the statistic errors, there is no differences among annihilations on $\mathrm{p}$ (events with $\mathrm{d}$ and one $p$ ) and annihilations on $n$ (events with $2 p$ ); 2) the like pion pair distributions are much flatter than the previous ones, which reflects in smaller values of $\gamma$, close to 1.2 for all sets of events.

The contribution to $\gamma^{++}$and $\gamma^{-}$comes out from events with at least two $\pi^{+}$or two $\pi^{-}$; that to $\gamma^{+-}$comes out from events with at least one $\pi^{+} \pi^{-}$pair. Hence, considering the combinations of numbers of $\pi^{+}$and $\pi^{-}$in Tab. 4.5 and the percentages in Tab. 4.4, we carry out that, for annihilations on $p$, the main contribution to $\gamma^{++}$and $\gamma^{-}$comes out from the final states $\left(2 \pi^{+} 2 \pi^{-} \mathrm{m} \pi^{0}\right)$ and $\left(1 \pi^{+} 1 \pi^{-} \mathrm{m} \pi^{0}\right)$. For the annihilations on $\mathrm{n}$, the main contribution to $\gamma^{++}$comes out from $\left(2 \pi^{+} 3 \pi^{-} \mathrm{m} \pi^{0}\right)$, to $\gamma^{-}$from $\left(\pi^{+} 2 \pi^{-} \mathrm{m} \pi^{\mathrm{o}}\right)$ and to $\gamma^{+-}$from both final states.

\subsection{Correlation between pions and heavy particles}

Fig. 6.2 shows the angular correlations between the $\pi$ and the proton and the deuteron. Here $\theta$ is the angle between a pion and heavy particle. The values of the quantity $\gamma$ defined as

$$
\gamma=\frac{\text { Number of tracks with } \theta>90^{\circ}}{\text { Number of tracks with } \theta<90^{\circ}}
$$

are shown in Tab. 6.2. We see that the pions are ejected with a small preference in the direction opposite to the deuteron, while are emitted symmetrically with respect to the proton direction. Presumably this symmetry is related to the presence of the neutron among the unseen uncharged particles.

\subsection{Comments}

The data from ${ }^{3} \mathrm{He}$ can be compared to those from ${ }^{1} \mathrm{H},{ }^{2} \mathrm{H}$ and ${ }^{4} \mathrm{He}$ which are shown in the same Tab. 6.1. The ${ }^{1} \mathrm{H}$ and ${ }^{2} \mathrm{H}$ data are not completely homogeneous to the ${ }^{3} \mathrm{He}$ ones as in the latter all channels with any number of charged and neutral pions are included, while for ${ }^{1} \mathrm{H}$ and ${ }^{2} \mathrm{H}$ only specific channels are known and only two values are at rest (see a review of data in Ref. 44). In the table, for the sake of simplicity, only the known channels with the highest branching ratio at 0 and about $1.2 \mathrm{MeV} / \mathrm{c}$ are shown. These channels contribute to those dominant in ${ }^{3} \mathrm{He}$. We note that there is no heavy change of the $\gamma$ values varying the antiproton momentum below $1 \mathrm{GeV} / \mathrm{c}$ and that $\gamma$ decreases as the number of pions increases. We see that the $\gamma$ values from the different nuclei are equal within the statistical errors. So no difference depending on the mass number is put in evidence by this comparison.

\section{CONCLUSION}

In this paper we give for the first time detailed information on the annihilation into pions of antiprotons at rest on ${ }^{3} \mathrm{He}$ nuclei together with some new data on ${ }^{4} \mathrm{He}$.

The data ( $\pi$ multiplicities for annihilation on single nucleons, $\pi N$ charge exchange, $\pi^{-}$ momentum distribution, two pion angular correlations) show that the annihilation on ${ }^{3} \mathrm{He}$ has features equal (within the statistical errors) to those on ${ }^{4} \mathrm{He}$, although the sizes of the two nuclei, the nucleon bonds and numbers are quite different.

Compared to the data from liquid hydrogen and deuterium, the $\pi^{-}$spectrum is a bit shifted towards the smaller momentum region, which is according to a weak final state interaction and to the dominance of the annihilation on proton over that on neutron. The $\pi \pi$ angular correlations are the same as on ${ }^{1} \mathrm{H}$ and the angular correlations for annihilation on proton are the same as on neutron. 
The $\pi^{-}$momentum distribution for annihilation on one proton shows a slight change going from ${ }^{1} \mathrm{H}$ to ${ }^{4} \mathrm{He}$, but the mean number of $\pi^{-}$is equal for the four nuclei considered (the latter statement holds also for the annihilation on one neutron), indicating that the $\pi$ absorption is negligible. The incidence of the $\pi \mathrm{N}$ charge exchange is revealed by the presence of 0 -heavy prong events, the probability of which amounts to $3.75 \%$, as on ${ }^{4} \mathrm{He}$.

Concluding, the comparison among data of pionic annihilation on ${ }^{1} \mathrm{H},{ }^{2} \mathrm{H},{ }^{3} \mathrm{He}$ and ${ }^{4} \mathrm{He}$ does not reveal, within the limits of our statistics, relevant effects due to the increase of the nucleon number in the annihilation region; the small differences can be seen as due to a weak final state interaction.

Acknowledgments. Thanks are due to Mr. Claudio Casella (Pavia) who applied himself to the task of scanning and measuring with praiseworthy care. 


\section{REFERENCES}

37 T.D. Lee and C.N. Yang, Nuovo Cimento 3 (1956) 749.

38 J. Riedelberger et al., Nucl. Phys. B (Proc. Suppl.) 8 (1989) 288.

39 G. Gregory et al., Nucl. Phys. B102 (1976) 189.

$40 \mathrm{~J}$. Roy, Antiproton-deuterium annihilation at rest, in IV International Symposium on antinucleon-nucleon interaction (Syracuse, 1975), p. III.1.

41 G.A. Smith, Pennsylvania State University Report PSU HEP/87-14 (1987).

42 V.V. Balashov et al., Sov. J. Nucl. Phys. 47 (1988) 1103.

43 G. Goldhaber et al., Phys. Rev. Lett. 3 (1959) 81.

44 R. Weill et al., Proceedings of the $4^{\text {th }}$ European antiproton symposium (Barr, Strasbourg, 1978), vol. 2. p. 147, Edition du CNRS, Paris (1979).

45 J. Bartke, Phys. Lett. B174 (1986) 32. 


\section{TABLE CAPTIONS}

3.1 Reactions on ${ }^{3} \mathrm{He}$ with initial production of 1 and 2 charged pions in antiproton-nucleon annihilation. $\pi \mathrm{N}$ charge exchange effects are shown separately. In square brackets the different charge exchange reactions are indicated.

4.1 Numbers of positive particles identified univocally $\left(\pi^{+}, p, d\right)$, of particles identified partially $\left(\pi^{+} \mathrm{p}\right.$ or $\left.\mathrm{pd}\right)$ and of particles totally unidentified $\left(\pi^{+} \mathrm{pd}\right) . \mathrm{K}^{+}$are included into $\pi^{+}$or p.

4.2 Numbers of events identified on the basis of the number $n_{h}$ of heavy prongs per events as a function of the multiplicities $M$ and $n_{\pi}$.

4.3 One heavy prong events given in Tab. 4.2 subdivided into events with an identified proton, an identified deuteron and an unidentified heavy particle ( $p$ or d).

4.4 (a) Percentages of events as a function of $M$ and $n_{h}$; (b) percentages as a function of $M$ only; (c) percentages as in (a) but normalized with respect to each value of $n_{h} . P=$ percentages of different types of reactions. $\langle x\rangle=$ mean value per event of the indicated quantities; $\left\langle\mathrm{n}_{\mathrm{h}}>\right.$ is given by $\left\langle\mathrm{M}><\mathrm{n}_{\pi^{-}}>-<\mathrm{n}_{\pi^{+}}\right\rangle$. The sum of the percentages in (a) should give those in column (b); there are, however, small differences due to the fact that column (b) comes out directly from a higher statistics (3017 events).

4.5 Number of $\pi^{+}$per event as a function of $M$ and of $n_{h}$.

4.6 $\pi^{+}$multiplicity distribution and mean number of $\pi^{+}$per event.

4.7 $\pi^{+}$and $\pi^{-}$multiplicity distributions for annihilation on single nucleons from different nuclei. $\left\langle\mathrm{n}_{\pi^{+}}>\right.$and $<\mathrm{n}_{\pi^{-}}>$are mean numbers of $\pi^{+}$and $\pi^{-}$per event. $\mathrm{H}^{1}$ from Ref. 35 , ${ }^{2} \mathrm{H}$ from Ref. $17,{ }^{4} \mathrm{He}$ from Ref. 15.

5.1 Temperature of the $\pi^{ \pm}$spectrum from different nuclei for annihilation at rest, assuming that the spectrum obeys to a maxwellian low.

(*) These data concern $\pi^{-}$only.

6.1 Angular correlation between like and unlike charged pions. The hydrogen and deuterium (neutron) data are taken from [40]. In square brackets the branching ratios for the different reactions ${ }^{[11]}$ and in round brackets the values of $\gamma^{-}$are given. For the definition of $\gamma$ see text.

6.2 Angular correlation for pion-proton and pion-deuteron pairs. For the definition of $\gamma \mathrm{see}$ text. 


\section{FIGURE CAPTIONS}

4.1 Stopping power of different particles in a gaseous helium target at normal conditions of temperature and pressure as a function of the momentum $(\mathrm{MeV} / \mathrm{c})$. The stopping power of $200 \mathrm{MeV} / \mathrm{c}$ protons (antiprotons) is assumed to be $100 \%$.

5.1 Full line: isotropy of the $\pi^{-}$angular distribution; $\lambda$ is the dip angle. Dashed line: angular distribution of $\pi^{-}$with measured momentum; the minima at $\sin \lambda= \pm 1$ correspond to tracks directed close to the magnetic field direction.

5.2 Momentum distributions of all $\pi^{-}$(full line) and of $\pi^{-}$tracks with dip angle within $\pm 30^{\circ}$ (dotted line).

$5.3 \pi^{-}$momentum distributions for different multiplicities $M\left(|\lambda| \leq 30^{\circ}\right)$.

5.4 Momentum distributions of $\pi^{-}$produced in events with a final $d$ and with two final $p$ to be compared with the all $\pi^{-}$distribution.

5.5 Momentum distribution of $\pi^{-}$produced in events without heavy prongs (dotted line) compared with the all $\pi^{-}$distribution.

5.6 Momentum distribution of all positive tracks.

5.7 Contribution to the momentum distribution of the positive particles by identified $\pi^{+}$ (hystogram), identified $\mathrm{p}(\mathrm{o})$, identified $\mathrm{d}(\bullet)$, unidentified heavy prongs ( $\mathrm{p}$ or $\mathrm{d},(\Delta)$ ), unidentified positive prongs $(\pi$ or $p$ or $d,(*))$.

$5.8 \pi^{+}$momentum distribution compared to that of $\pi^{-}\left(|\lambda| \leq 30^{\circ}\right)$.

5.9 Comparison among the $\pi^{-}$spectra from ${ }^{3} \mathrm{He}$ (full line), ${ }^{1} \mathrm{H}$ (dashed line) and $\mathrm{n}\left({ }^{2} \mathrm{H}\right)$ (dotted line). ${ }^{1} \mathrm{H}$ and ${ }^{2} \mathrm{H}$ data are taken from [40].

5.10 Comparison among the $\pi^{-}$spectra from ${ }^{3} \mathrm{He}$ (full line), ${ }^{4} \mathrm{H}$ (stars) and ${ }^{20} \mathrm{Ne}$ (dashed line). For all the data $|\lambda| \leq 30^{\circ}$.

5.11 Fits on the $\pi^{-}$momentum distributions from ${ }^{3} \mathrm{He},{ }^{4} \mathrm{He}$ and ${ }^{20} \mathrm{Ne}$ by a maxwellian function. The reduced $\chi^{2}$ values are, respectively, 1.91, 1.85 and 1.67.

6.1 Correlation between like and unlike charge pion pairs. $\cos \theta$ distributions for $\pi^{+} \pi^{+}$, $\pi^{-} \pi^{-}$and $\pi^{+} \pi^{-}$pairs. $\theta$ is the angle between two $\pi$ tracks of the same event.

6.2 Angular correlations for pion-proton and pion-deuteron pairs. 
TABLE 3.1

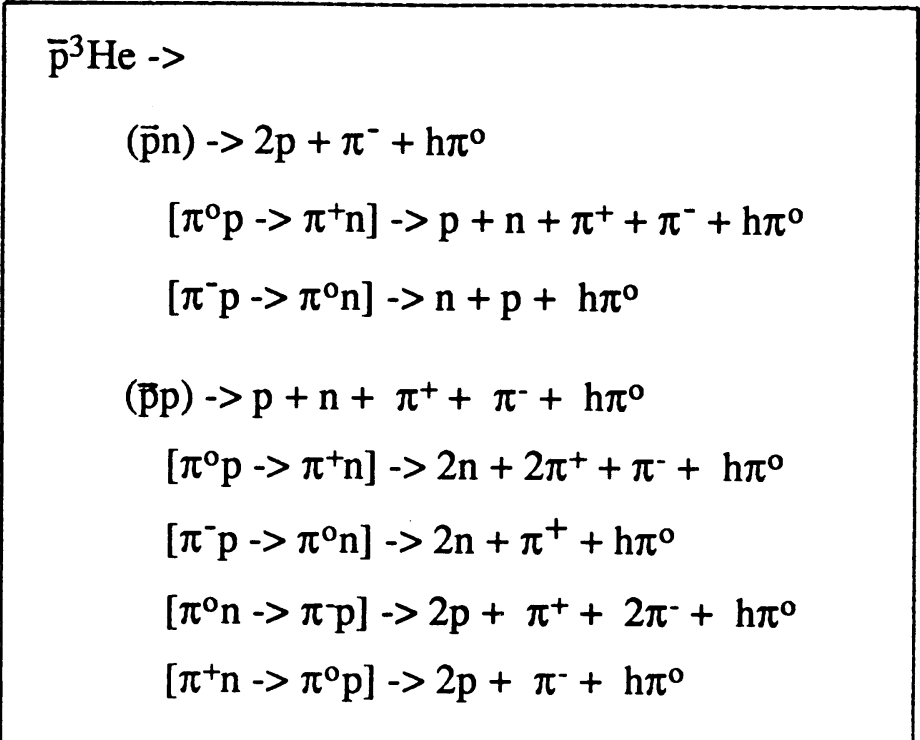

TABLE 4.1

\begin{tabular}{|rrr|}
\hline$\pi^{+}=1718$ & $\pi^{+} \mathrm{p}=742$ & $\pi^{+} \mathrm{pd}=796$ \\
& $\mathrm{p}=739$ & $\mathrm{pd}=354$ \\
& & $\mathrm{~d}=269$ \\
Tot $=4618$ & & \\
\hline
\end{tabular}

TABLE 4.2

\begin{tabular}{|c|c|c|c|c|c|c|c|c|}
\hline & & & & & & & & \\
\hline M & $\mathrm{n}_{\pi^{-}}$ & 0 & 1 & $\leq 1$ & $\geq 1$ & 2 & $0,1,2$ & Tot. \\
\hline 1 & 0 & 7 & 51 & 41 & --- & --. & $\cdots$ & 99 \\
\hline 3 & 1 & 18 & 214 & 167 & 193 & 29 & 172 & 793 \\
\hline 5 & 2 & 12 & 204 & 72 & 314 & 98 & 281 & 981 \\
\hline 7 & 3 & 1 & 14 & 7 & 63 & 25 & 34 & 144 \\
\hline 9 & 4 & --- & --- & -- & 1 & 2 & --- & 3 \\
\hline & & 38 & 483 & 287 & 571 & 154 & 521 & 2020 \\
\hline
\end{tabular}


TABLE 4.3

\begin{tabular}{|ccrrr|}
\hline $\mathrm{M}$ & $\mathrm{n}_{\pi^{-}}$ & $\mathrm{p}$ & $\mathrm{d}$ & $\mathrm{pd}$ \\
\hline 1 & 0 & 15 & 15 & 21 \\
3 & 1 & 62 & 87 & 65 \\
5 & 3 & 54 & 100 & 50 \\
7 & 4 & 4 & 8 & 2 \\
\hline \multicolumn{2}{|c}{ Tot. } & 135 & 210 & 138 \\
\hline
\end{tabular}

TABLE 4.4

\begin{tabular}{|c|c|c|c|c|c|c|c|}
\hline \multirow[b]{2}{*}{$M n_{\pi}$} & \multicolumn{3}{|c|}{$\begin{array}{l}\text { (a) } \\
\mathrm{n}_{\mathrm{h}}\end{array}$} & \multicolumn{2}{|l|}{ (b) } & \multicolumn{2}{|l|}{ (c) } \\
\hline & 0 & 1 & 2 & Tot. & 0 & 1 & 2 \\
\hline 0 & $0.59 \pm 0.22$ & $4.31 \pm 0.47$ & & $5.14 \pm 0.40$ & 15.75 & 5.57 & \\
\hline 1 & $1.95 \pm 0.46$ & $34.58 \pm 1.80$ & $2.72 \pm 0.50$ & $39.38 \pm 0.88$ & 52.07 & 44.69 & 14.42 \\
\hline 2 & $1.11 \pm 0.32$ & $35.31 \pm 2.15$ & $12.13 \pm 1.22$ & $48.22 \pm 0.91$ & 29.64 & 45.64 & 64.31 \\
\hline 3 & $0.09 \pm 0.09$ & $3.17 \pm 0.70$ & $3.86 \pm 0.77$ & $7.06 \pm 0.46$ & 2.54 & 4.10 & 20.46 \\
\hline 4 & & & $0.15 \pm 0.11$ & $0.19 \pm 0.08$ & & & 0.79 \\
\hline $\mathrm{P}$ (Tot.) & $3.74 \pm 0.68$ & $77.37 \pm 2.93$ & $18.86 \pm 1.53$ & 100 & 100 & 100 & 100 \\
\hline $\mathrm{P}(1 \mathrm{p})$ & & 30.27 & & & & & \\
\hline $\mathrm{P}(\mathrm{d})$ & & 47.10 & & & & & \\
\hline$<\mathrm{n}_{\pi^{-}}>$ & $1.19 \pm 0.22$ & $1.48 \pm 0.07$ & $2.08 \pm 0.18$ & $1.578 \pm 0.025$ & & & \\
\hline$<\mathrm{M}>$ & $3.38 \pm 0.59$ & $3.96 \pm 0.05$ & $5.15 \pm 0.44$ & $4.155 \pm 0.062$ & & & \\
\hline$<\mathrm{n}_{\mathrm{h}}>$ & & & & $1.145 \pm 0.083$ & & & \\
\hline
\end{tabular}

TABLE 4.5

\begin{tabular}{|ccccc|}
\hline & \multicolumn{5}{c}{$\mathrm{n}_{\mathrm{h}}$} \\
$\mathrm{M}$ & $\mathrm{n}_{\pi^{-}}$ & 0 & 1 & 2 \\
\hline 1 & 0 & 1 & 0 & --- \\
3 & 1 & 2 & 1 & 0 \\
5 & 2 & 3 & 2 & 1 \\
7 & 3 & 4 & 3 & 2 \\
9 & 4 & 5 & 4 & 3 \\
\hline
\end{tabular}


TABLE 4.6

\begin{tabular}{|cc|}
\hline $\mathrm{n}_{\pi^{-}}$ & $\mathrm{p}(\%)$ \\
\hline 0 & $7.03 \pm 0.78$ \\
1 & $47.30 \pm 2.66$ \\
2 & $41.12 \pm 2.63$ \\
3 & $4.43 \pm 0.91$ \\
4 & $0.095 \pm 0.095$ \\
\hline$<\mathrm{n}_{\pi^{+>}}$ & $1.432 \pm 0.065$ \\
\hline
\end{tabular}

TABLE 4.7

\begin{tabular}{|c|c|c|c|c|c|}
\hline $\mathrm{n}_{\pi^{-}}$ & $\mathrm{n}_{\pi^{+}}$ & ${ }^{1} \mathrm{H}$ & ${ }^{2} \mathrm{H}$ & ${ }^{3} \mathrm{He}$ & ${ }^{4} \mathrm{He}$ \\
\hline \multicolumn{6}{|c|}{$\overline{\mathrm{p} p}$ annihilation } \\
\hline 0 & 0 & $6.27 \pm 0.53$ & $6.4 \pm 0.4$ & $6.04 \pm 0.59$ & $4.96 \pm 0.52$ \\
\hline 1 & 1 & $42.38 \pm 2.37$ & $42.3 \pm 1.0$ & $45.15 \pm 1.38$ & $46.64 \pm 1.65$ \\
\hline 2 & 2 & $47.32 \pm 2.82$ & $46.9 \pm 1.1$ & $44.69 \pm 1.68$ & $43.83 \pm 2.54$ \\
\hline 3 & 3 & $4.02 \pm 0.64$ & $4.4 \pm 0.3$ & $4.12 \pm 0.71$ & $4.20 \pm 1.42$ \\
\hline 4 & 4 & & & & $0.35 \pm 0.15$ \\
\hline \multicolumn{2}{|c|}{$<\mathrm{n}_{\pi^{-}}>=<\mathrm{n}_{\pi^{+}}>$} & $1.49 \pm 0.06$ & $1.49 \pm 0.02$ & $1.47 \pm 0.04$ & $1.47 \pm 0.03$ \\
\hline $\mathrm{n}_{\pi^{-}}$ & $\mathrm{n}_{\pi^{+}}$ & & \multicolumn{2}{|c|}{$\overline{\mathrm{p}} \mathrm{n}$ annihilation } & \\
\hline 1 & 0 & & $16.8 \pm 0.8$ & $14.05 \pm 2.53$ & $21.50 \pm 2.54$ \\
\hline 2 & 1 & & $59.5 \pm 1.4$ & $65.14 \pm 5.86$ & $56.54 \pm 5.10$ \\
\hline 3 & 2 & & $23.0 \pm 0.9$ & $20.02 \pm 3.17$ & $21.37 \pm 3.18$ \\
\hline 4 & 3 & & $0.7 \pm 0.2$ & $0.79 \pm 0.47$ & $0.59 \pm 0.25$ \\
\hline \multicolumn{2}{|c|}{$<\mathrm{n}_{\pi^{-}}>$} & & $2.07 \pm 0.04$ & $2.07 \pm 0.15$ & $2.01 \pm 0.05$ \\
\hline \multicolumn{2}{|c|}{$<\mathrm{n}_{\pi^{+}}>$} & & 1.07 & 1.07 & 1.01 \\
\hline
\end{tabular}


TABLE 5.1

\begin{tabular}{|ccccccc|}
\hline Refs. & ${ }^{1} \mathrm{H}$ & ${ }^{2} \mathrm{H}$ & ${ }^{3} \mathrm{H}$ & ${ }^{4} \mathrm{H}$ & ${ }^{14} \mathrm{~N}$ & ${ }^{20} \mathrm{Ne}$ \\
\hline$[38]$ & $128 \pm 1$ & $126 \pm 1$ & & & $131 \pm 1$ & \\
{$[41]$} & & & & & $136 \pm 3$ & \\
This exp. & & & $135 \pm 3^{*}$ & $136 \pm 2^{*}$ & & $128 \pm 1 *$ \\
\hline
\end{tabular}

TABLE 6.1

\begin{tabular}{|c|c|c|c|}
\hline & $\gamma^{\#}$ & $\gamma^{+}$ & $\mathrm{P}(\mathrm{GeV} / \mathrm{c})$ \\
\hline${ }^{3} \mathrm{He}$ (all events) & $\begin{array}{c}1.21 \pm 0.04 \\
(1.25 \pm 0.07)\end{array}$ & $1.82 \pm 0.05$ & 0 \\
\hline (identified events) & $\begin{array}{c}1.09 \pm 0.06 \\
(1.21 \pm 0.09)\end{array}$ & $2.05 \pm 0.08$ & 0 \\
\hline${ }^{4} \mathrm{He}$ (all events) & $1.20 \pm 0.08$ & $1.82 \pm 0.10$ & 0 \\
\hline \multicolumn{4}{|l|}{${ }^{1} \mathrm{H}$} \\
\hline $2 \pi^{+} 2 \pi^{-}[7 \%]$ & $1.35 \pm 0.02$ & $3.50 \pm 0.05$ & 0 \\
\hline $2 \pi^{+} 2 \pi^{-} 1 \pi^{\circ}[19.7 \%]$ & $1.14 \pm 0.10$ & $2.26 \pm 0.15$ & 0 \\
\hline $2 \pi^{+} 2 \pi^{-} 1 \pi^{\circ}[19.7 \%]$ & $1.40 \pm 0.02$ & $2.14 \pm 0.03$ & 1.2 \\
\hline $2 \pi^{+} 2 \pi^{-} 2.5 \pi^{\circ}[40 \%]$ & $1.75 \pm 0.03$ & $1.75 \pm 0.04$ & 1.2 \\
\hline \multicolumn{4}{|l|}{$\mathrm{n}$} \\
\hline $2 \pi^{+} 3 \pi^{-}$ & $1.37 \pm 0.03$ & $2.43 \pm 0.03$ & $1-1.6$ \\
\hline
\end{tabular}

TABLE 6.2

\begin{tabular}{|cccc|}
\hline & \multicolumn{3}{c|}{$\gamma$} \\
& $\pi^{+}$ & $\pi^{-}$ & $\pi^{ \pm}$ \\
\hline $\mathrm{p}$ & $1.006 \pm 0.077$ & $1.006 \pm 0.056$ & $1.006 \pm 0.046$ \\
$\mathrm{~d}$ & $1.242 \pm 0.095$ & $1.206 \pm 0.091$ & $1.223 \pm 0.066$ \\
\hline
\end{tabular}




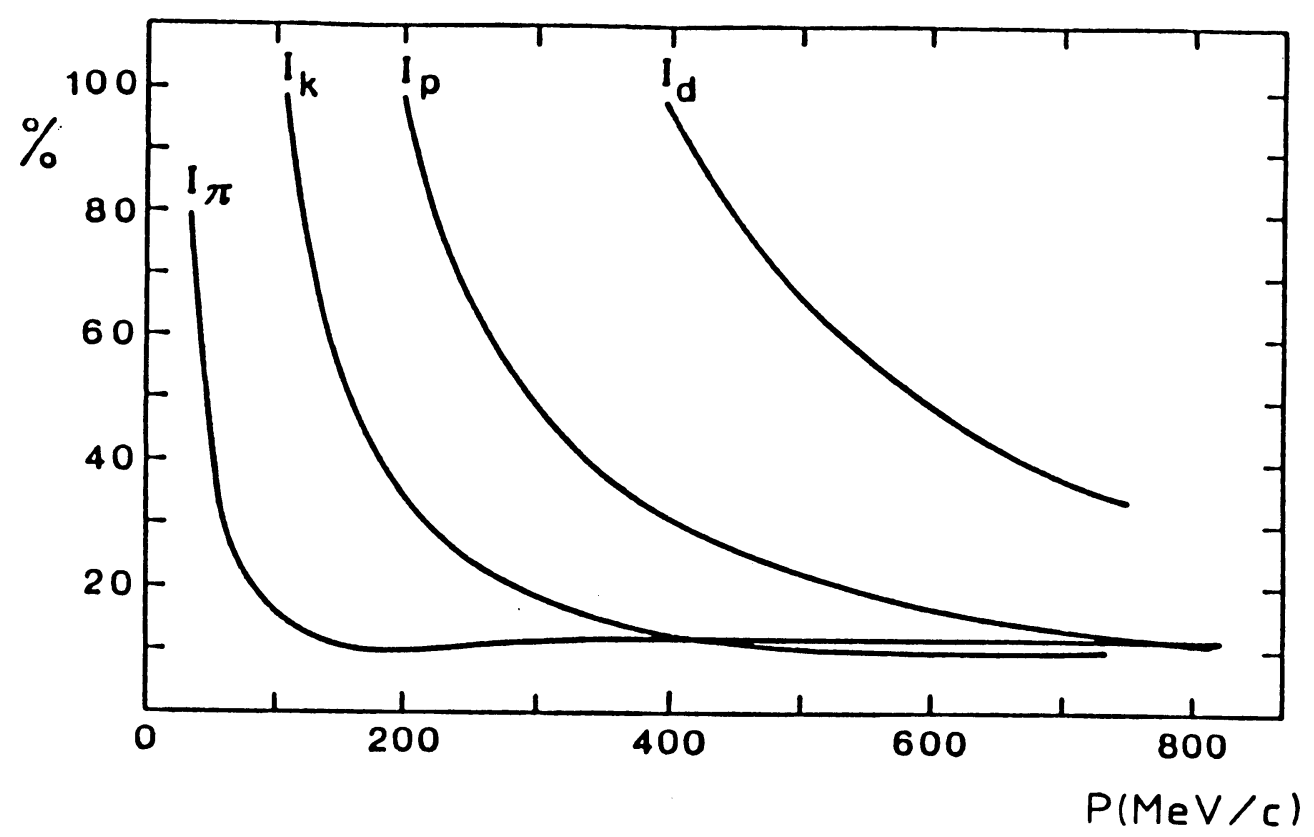

Fig. 4.1

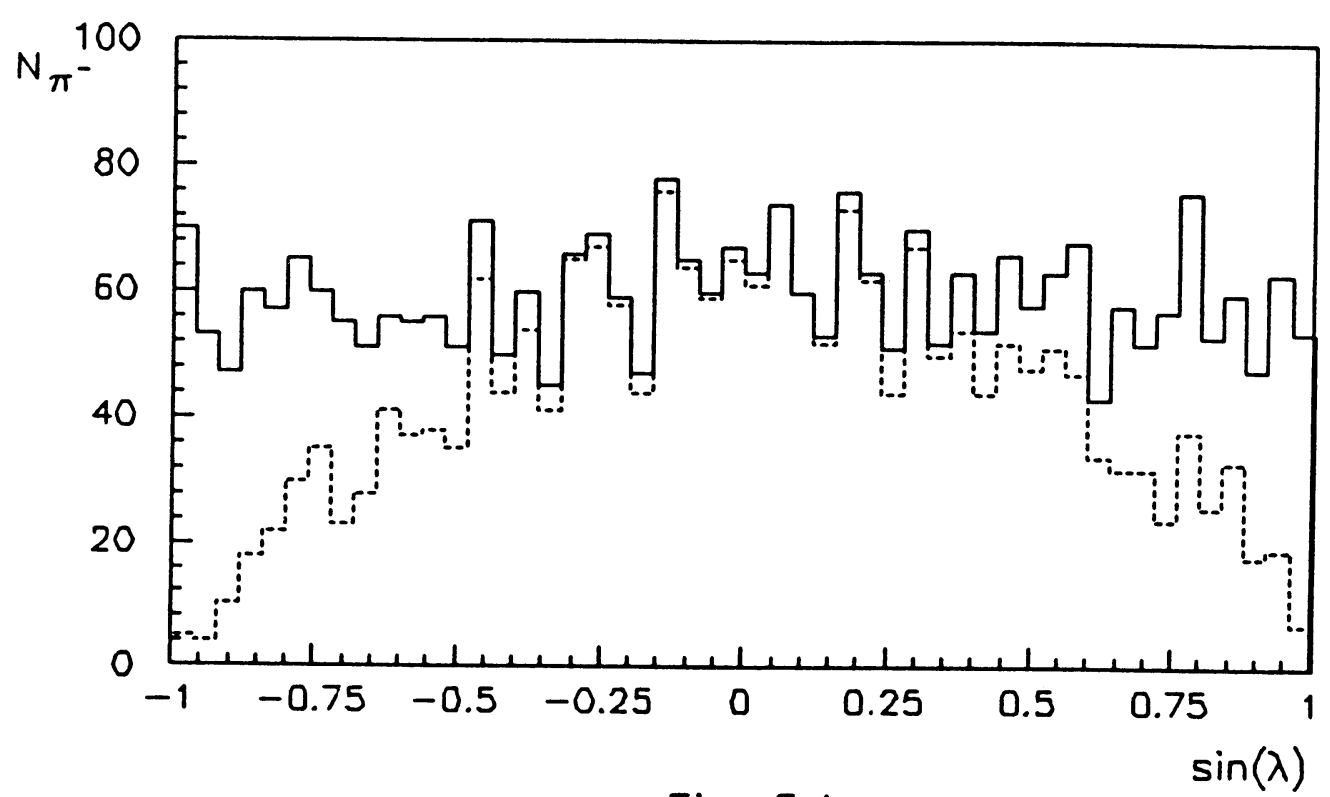

Fig. 5.1

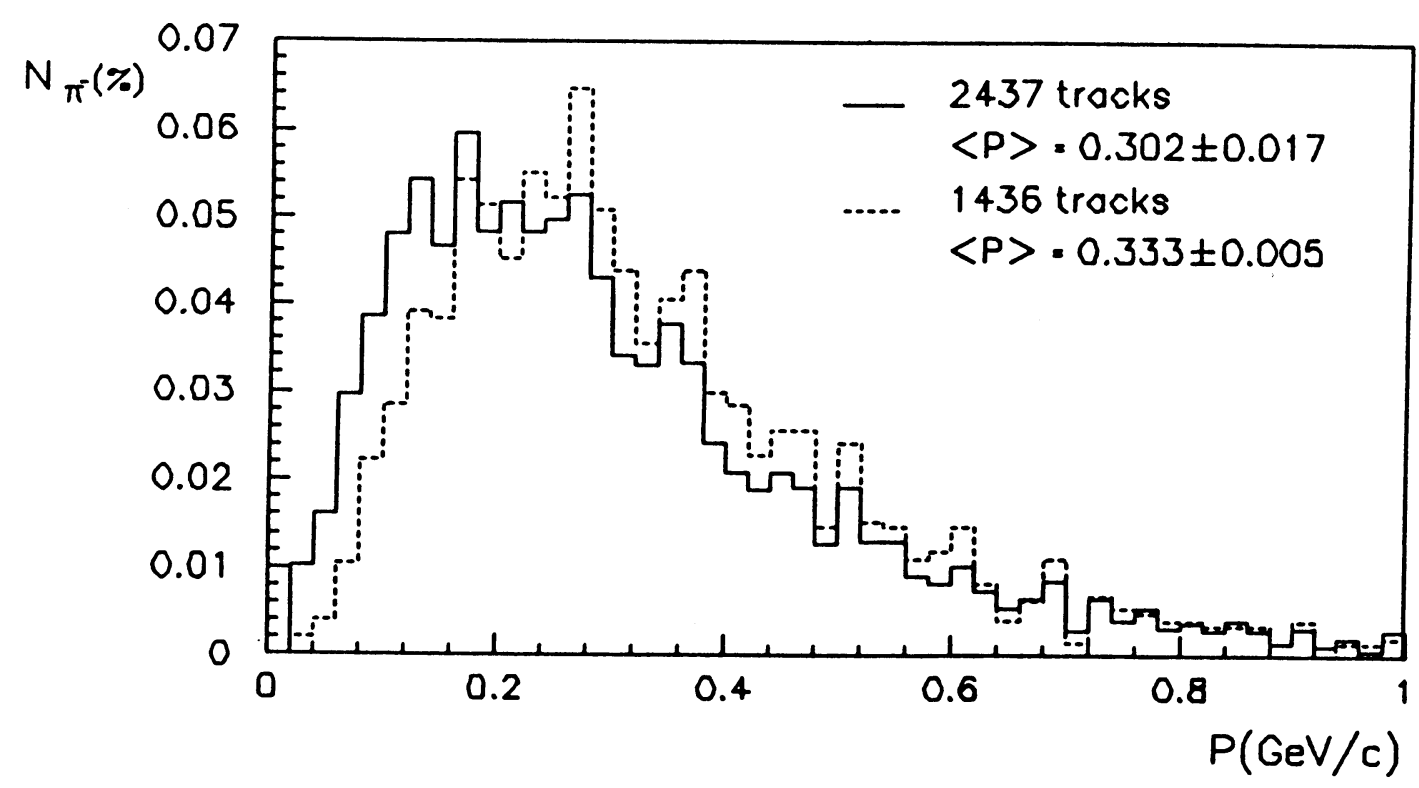

Fig. 5.2 


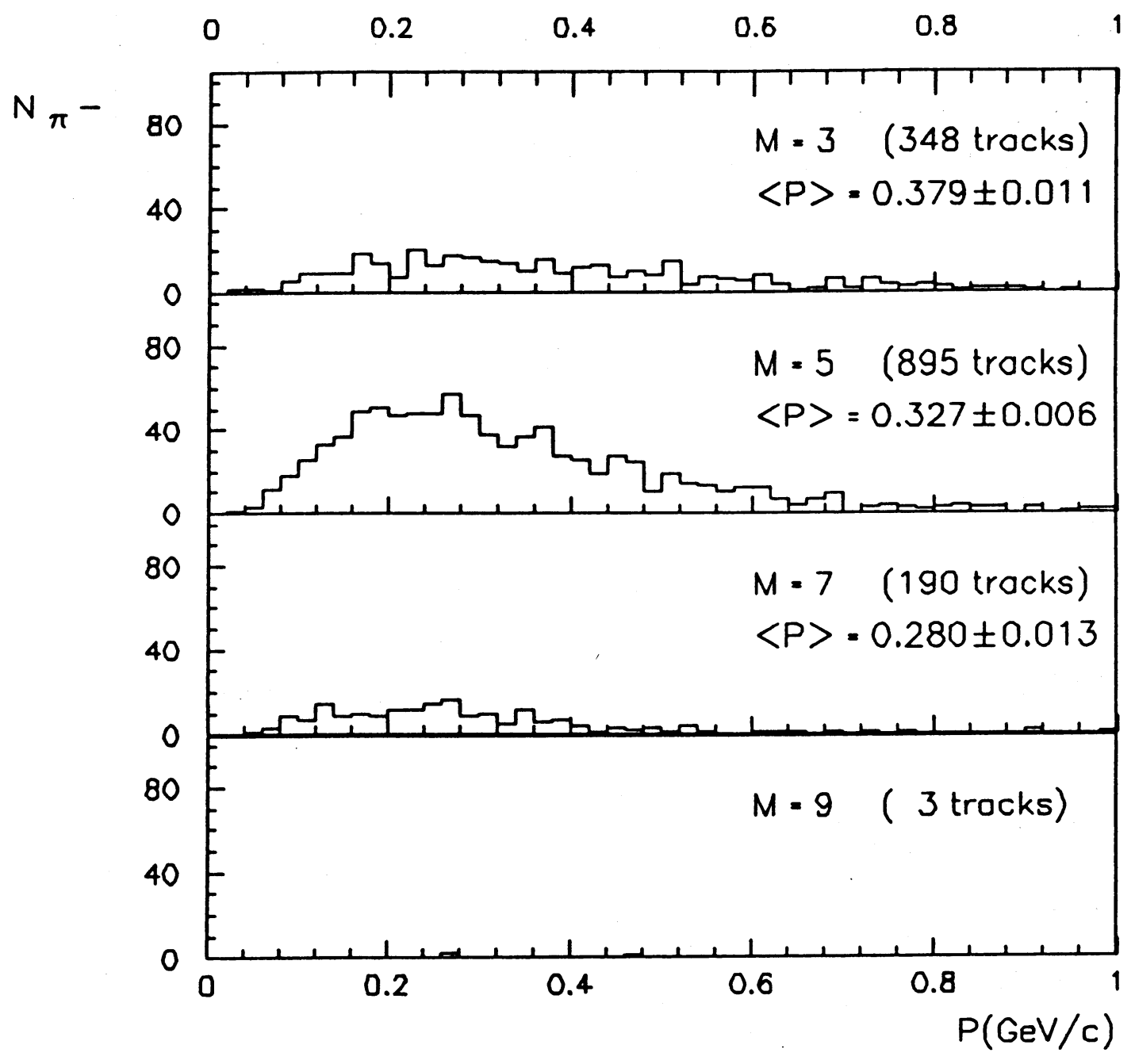

Fig. 5.3 


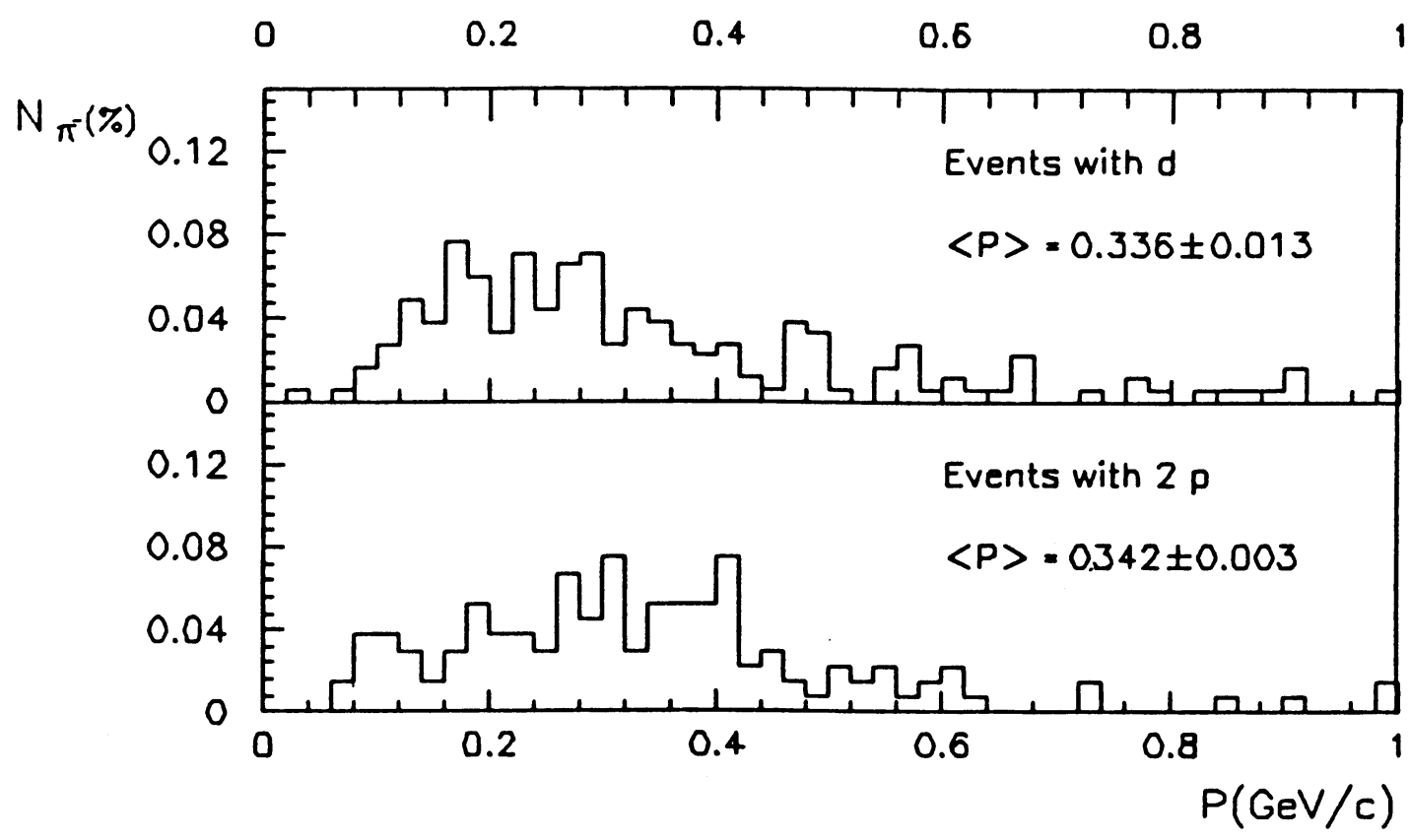

Fig. 5.4

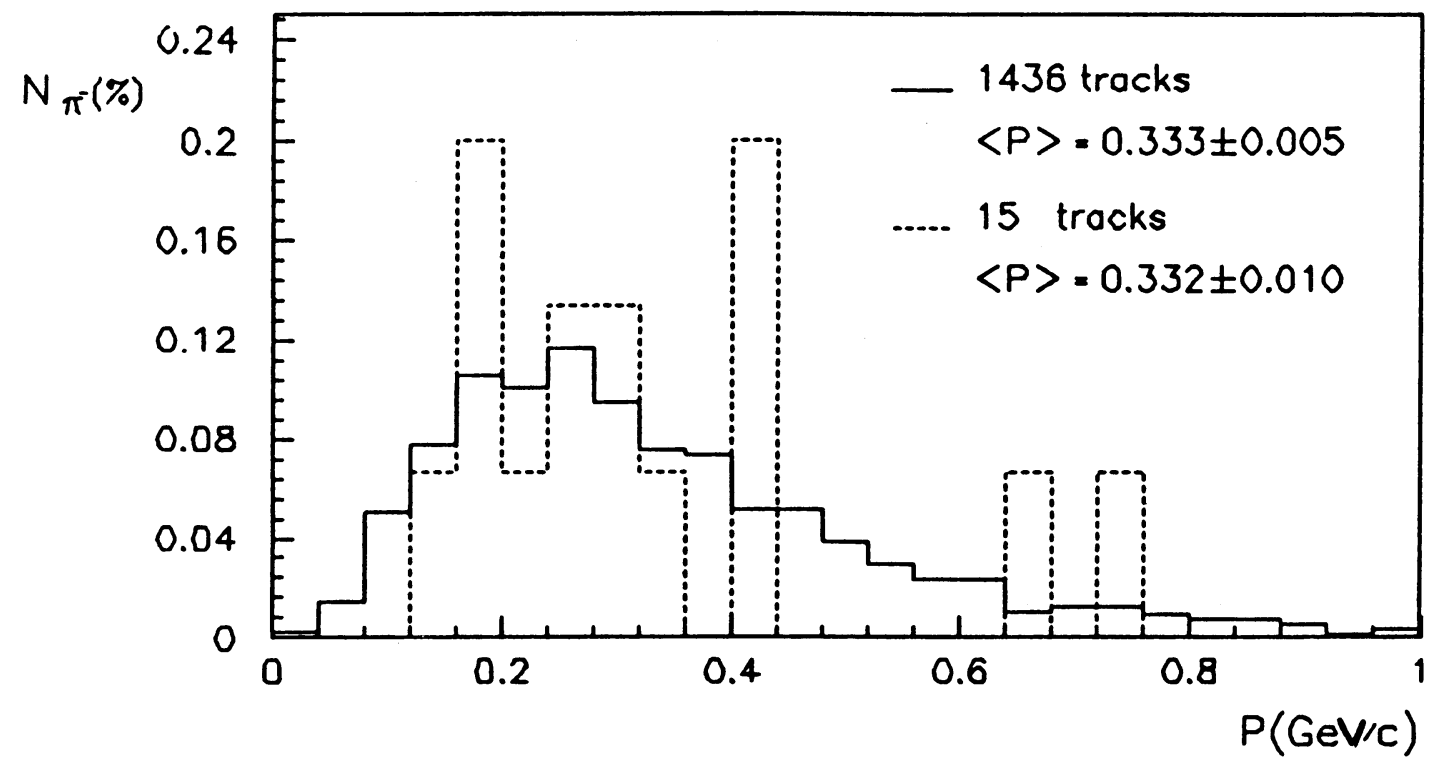

Fig. 5.5 


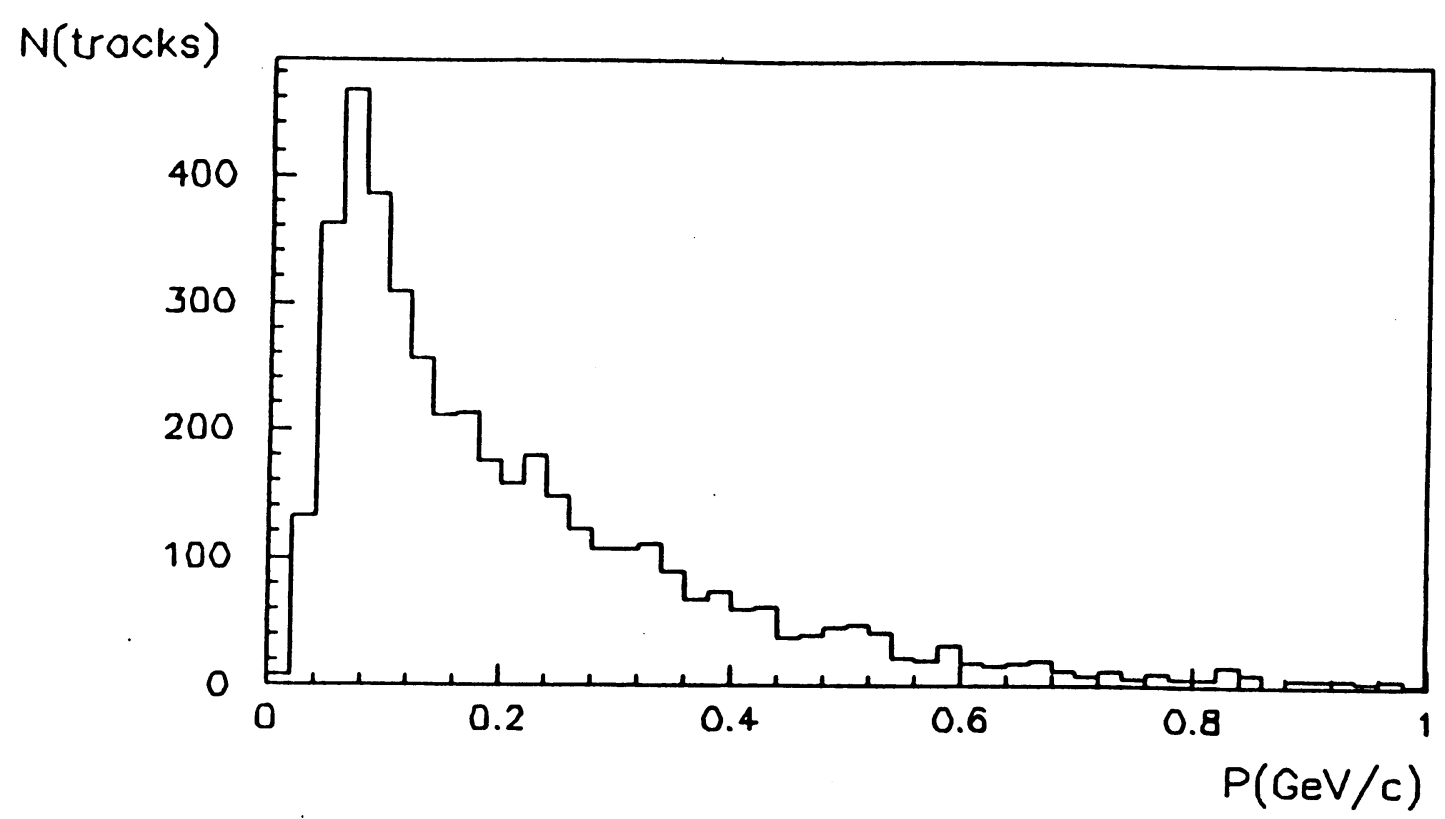

Fig. 5.6

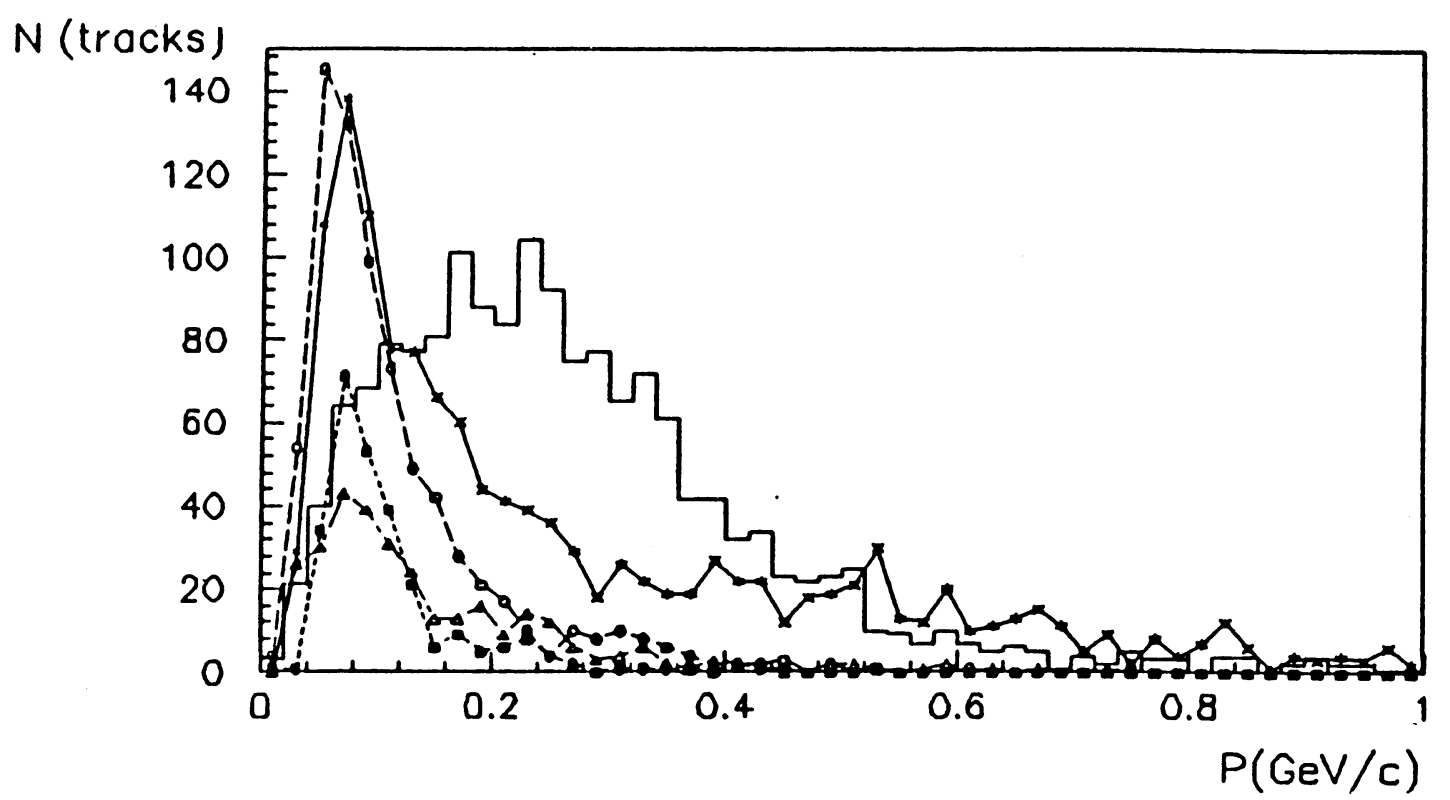

Fig. 5.7

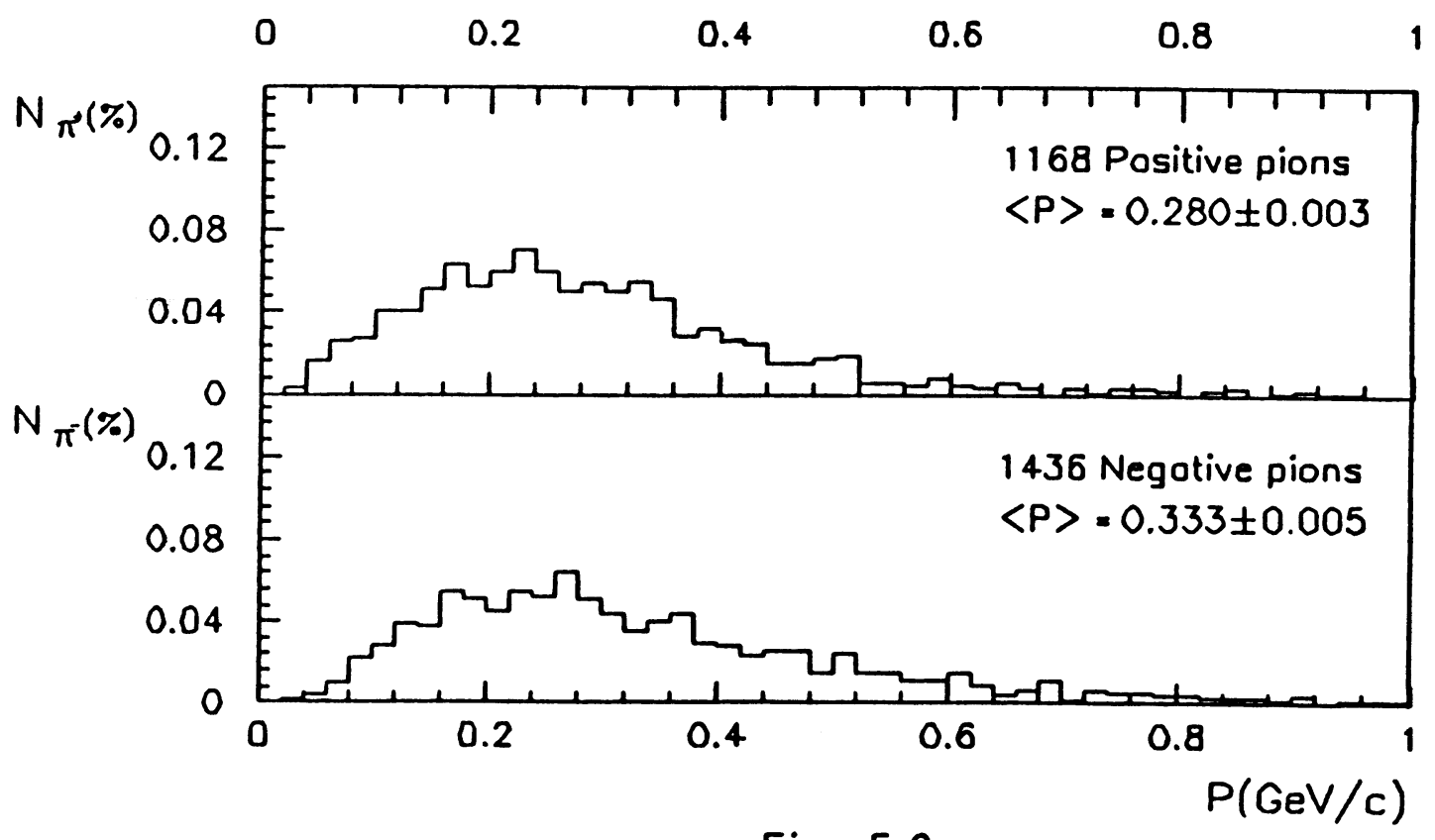

Fig. 5.8 


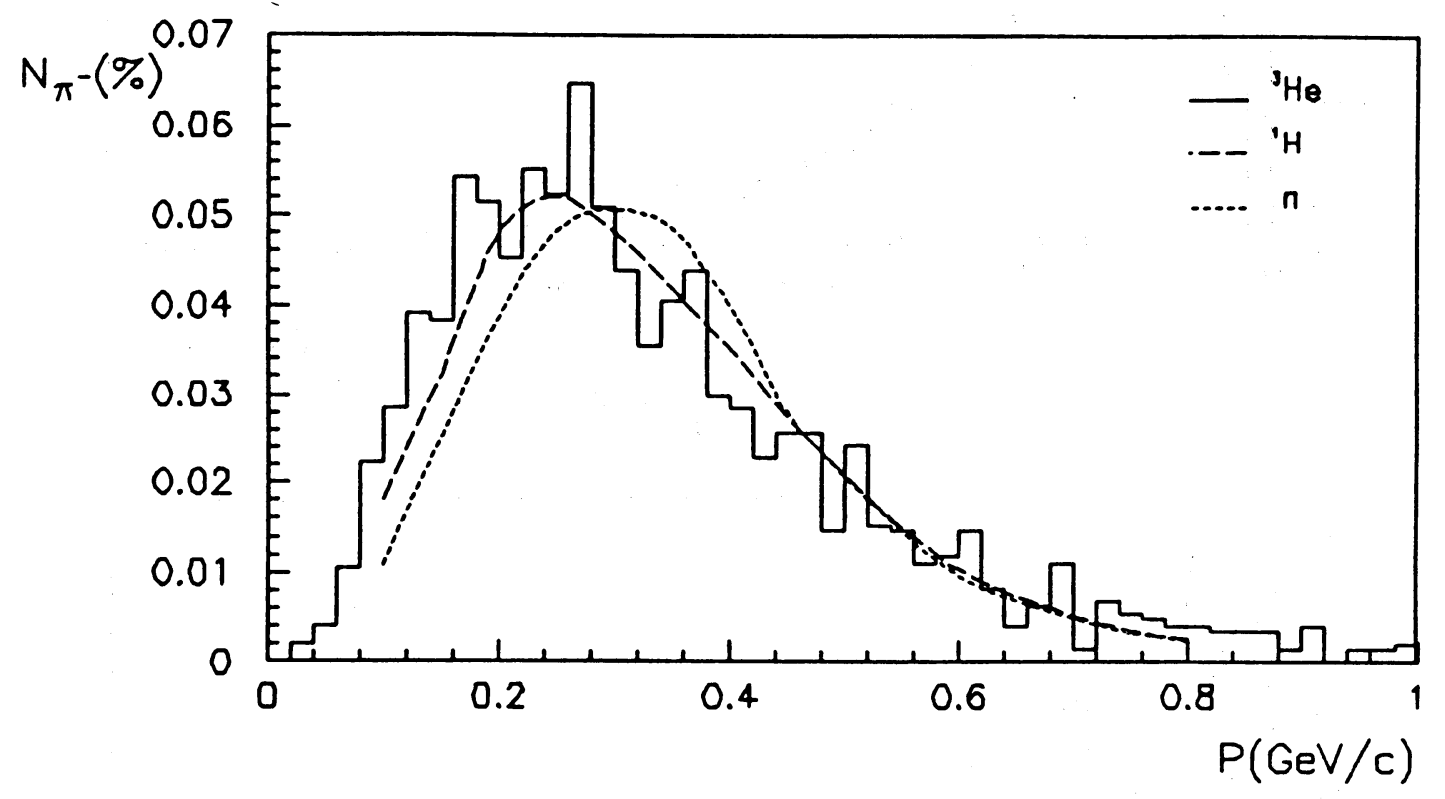

Fig. 5.9

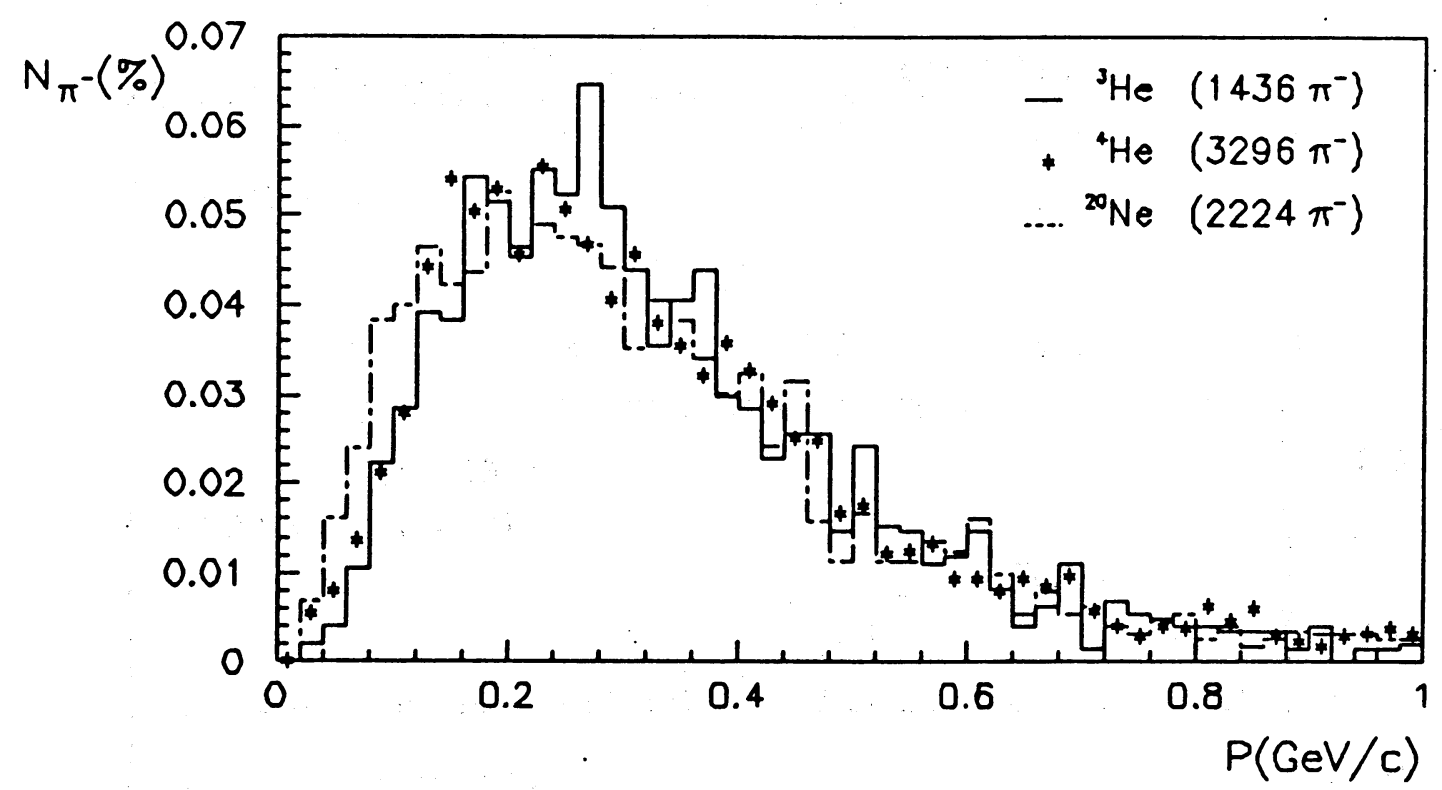

Fig. 5.10 


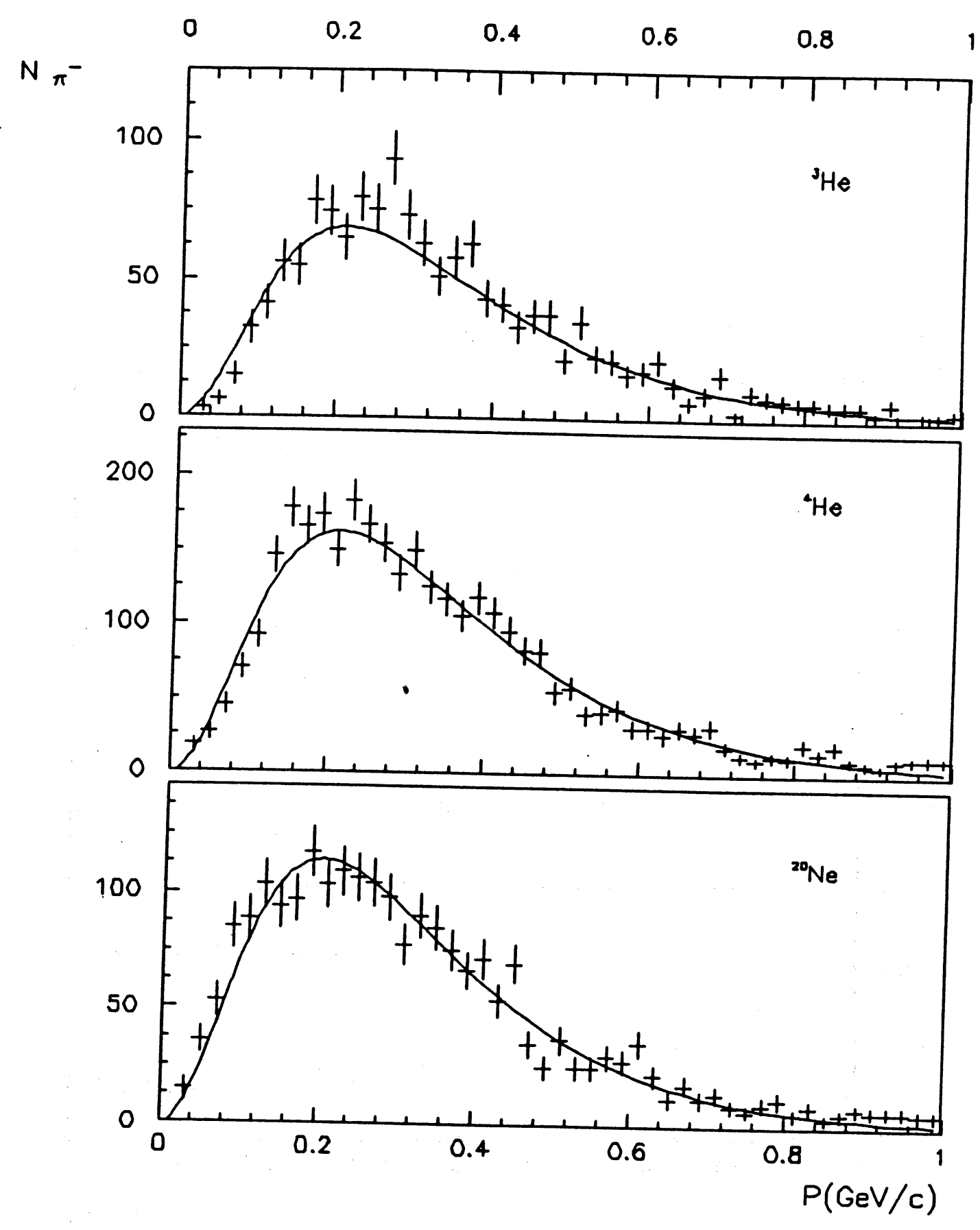

Fig. 5.11 


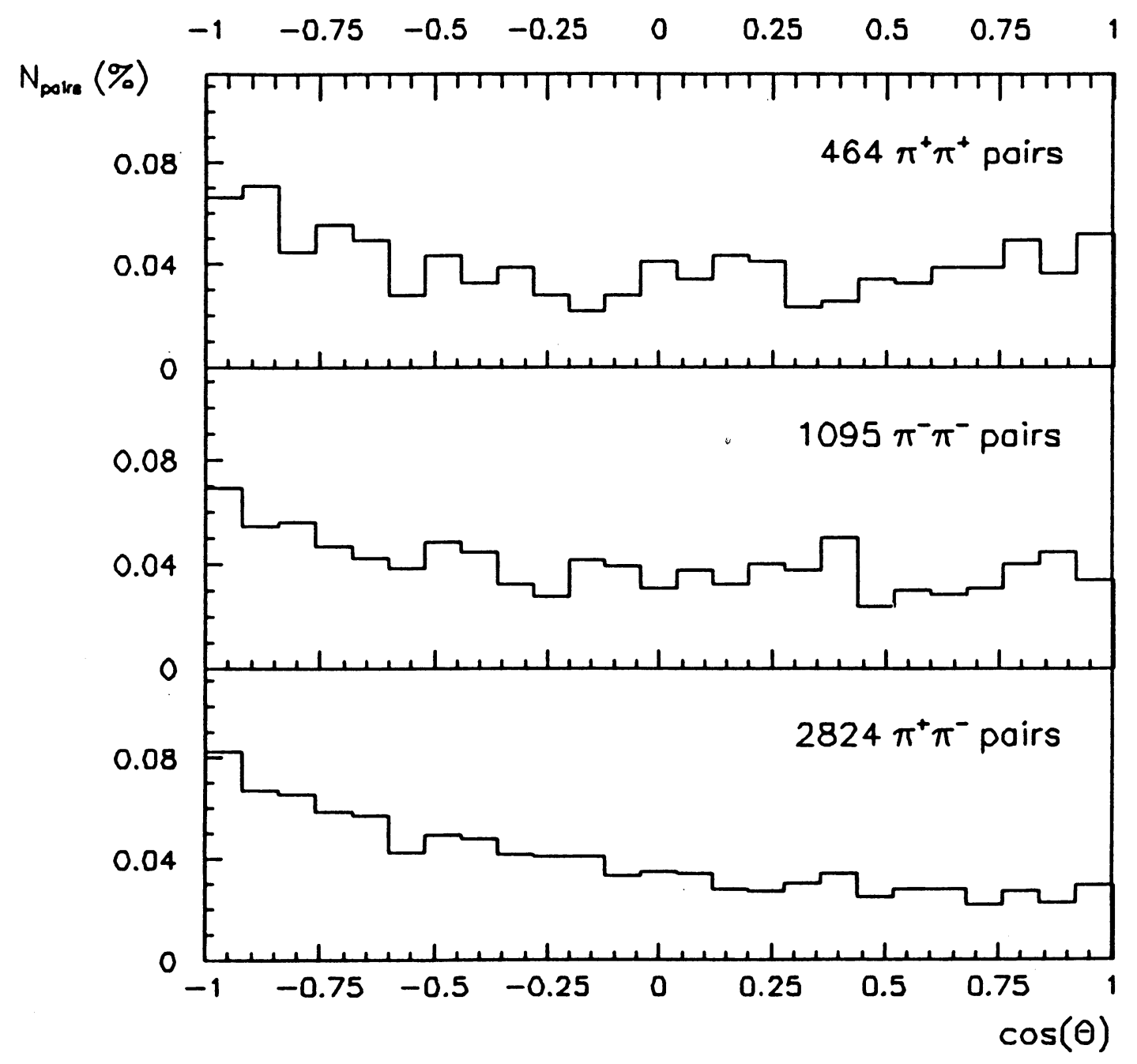

Fig. 6.1 


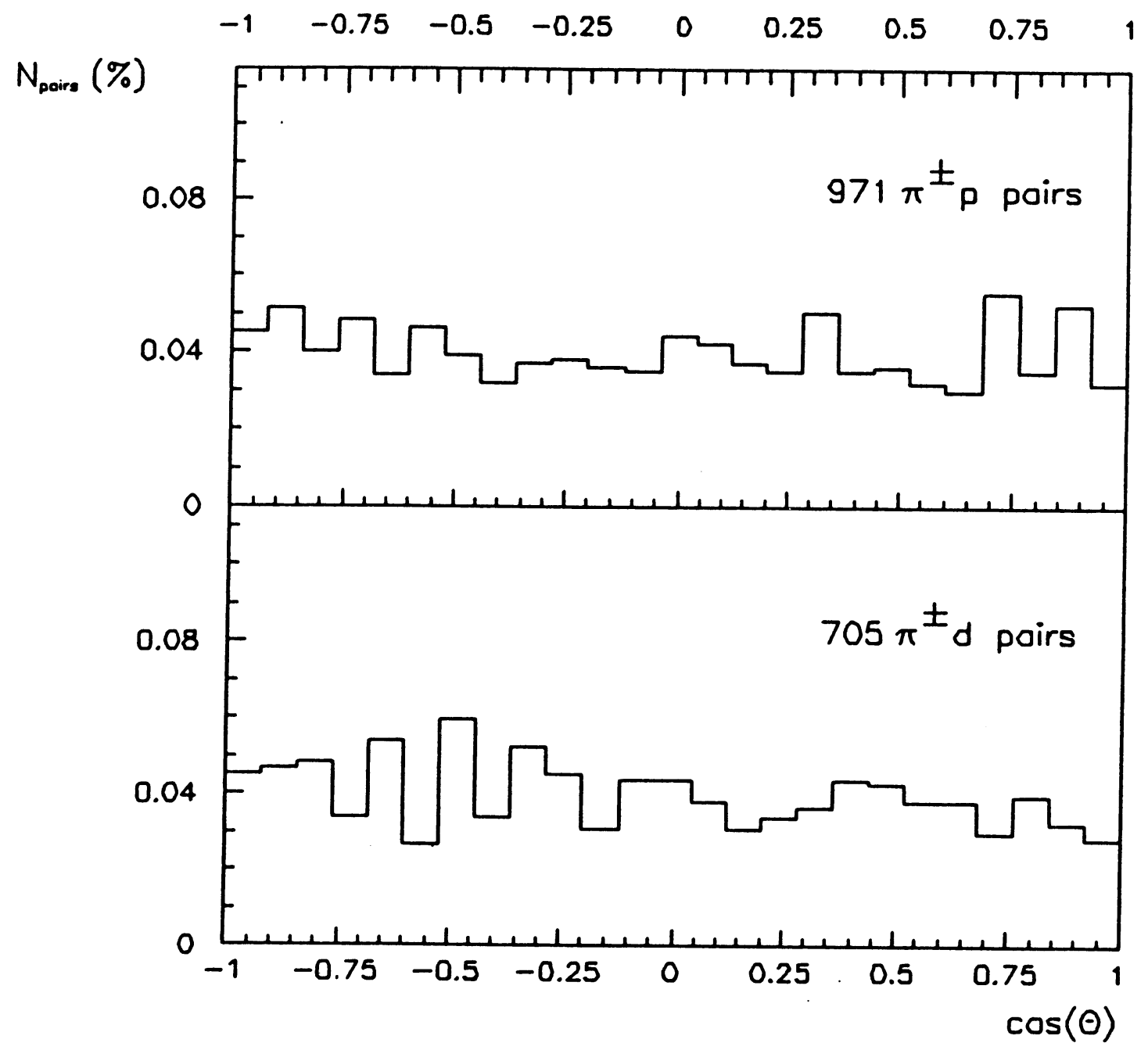

Fig. 6.2 